\title{
NONLINEAR ELLIPTIC ANISOTROPIC PROBLEM WITH NON-LOCAL BOUNDARY CONDITIONS AND $L^{1}$-DATA
}

\author{
ADAMA KABORÉ, STANISLAS OUARO* \\ ${ }^{1}$ Department of Mathematics, Laboratoire de Mathematiques et Informatiques (LAMI), UFR. Sciences Exactes et Appliquées, Université \\ Joseph KI-ZERBO, 03 BP 7021 Ouaga 03, Burkina Faso \\ Corresponding author: ouaro@yahoo.fr, souaro@univ-ouaga.bf
}

Received Nov. 13, 2019

\begin{abstract}
Aвstract. We study a nonlinear anisotropic elliptic problem with non-local boundary conditions and $L^{1}$-data. We prove an existence and uniqueness result of entropy solution.

2010 Mathematics Subject Classification. 35J05, 35J25, 35J60, 35J66.
\end{abstract}

Key words and phrases. entropy solution; non-local boundary conditions; Leray-Lions operator.

\section{InTRODUCTION}

Let $\Omega$ be a bounded domain in $\mathbb{R}^{N}(N \geq 2)$ such that $\partial \Omega$ is Lipschitz and $\partial \Omega=\Gamma_{D} \cup \Gamma_{N e}$ with $\Gamma_{D} \cap \Gamma_{N e}=\emptyset$. Our aim is to study the following problem.

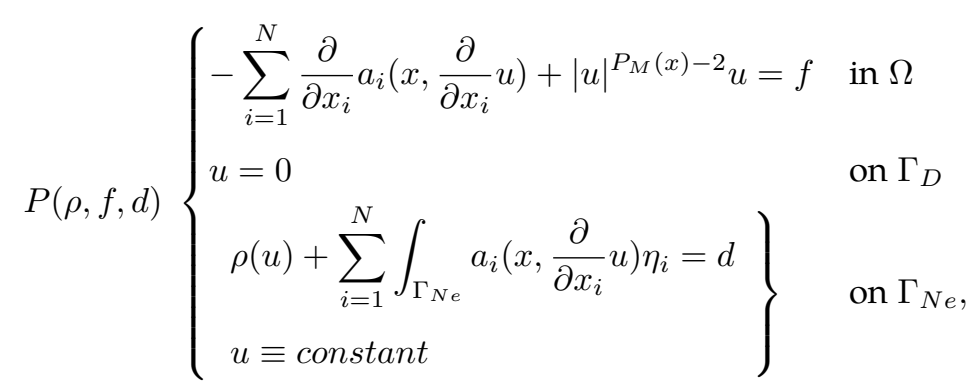

where the right-hand side $f \in L^{1}(\Omega)$ and $\eta_{i}, i \in\{1, \ldots, N\}$ are the components of the outer normal unit vector. For any $\Omega \subset \mathbb{R}^{N}$, we set

$$
C_{+}(\bar{\Omega})=\left\{h \in C(\bar{\Omega}): \inf _{x \in \Omega} h(x)>1\right\},
$$

and we denote

$$
h^{+}=\sup _{x \in \Omega} h(x), h^{-}=\inf _{x \in \Omega} h(x) .
$$

DOI: 10.28924/APJM/7-4 
For the exponents, $\vec{p}():. \bar{\Omega} \rightarrow \mathbb{R}^{N}, \vec{p}()=.\left(p_{1}(),. \ldots, p_{N}().\right)$ with $p_{i} \in C_{+}(\bar{\Omega})$ for every $i \in\{1, \ldots, N\}$ and for all $x \in \bar{\Omega}$. We put $p_{M}(x)=\max \left\{p_{1}(x), \ldots, p_{N}(x)\right\}$ and $p_{m}(x)=\min \left\{p_{1}(x), \ldots, p_{N}(x)\right\}$.

We assume that for $i=1, \ldots, N$, the function $a_{i}: \Omega \times \mathbb{R} \rightarrow \mathbb{R}$ is Carathéodory and satisfies the following conditions:

- $\left(H_{1}\right): a_{i}(x, \xi)$ is the continuous derivative with respect to $\xi$ of the mapping $A_{i}=A_{i}(x, \xi)$, that is, $a_{i}(x, \xi)=\frac{\partial}{\partial \xi} A_{i}(x, \xi)$ such that the following equality holds.

$$
A_{i}(x, 0)=0
$$

for almost every $x \in \Omega$.

- $\left(H_{2}\right)$ : There exists a positive constant $C_{1}$ such that

$$
\left|a_{i}(x, \xi)\right| \leq C_{1}\left(j_{i}(x)+|\xi|^{p_{i}(x)-1}\right),
$$

for almost every $x \in \Omega$ and for every $\xi \in \mathbb{R}$, where $j_{i}$ is a non-negative function in $L^{p_{i}^{\prime}(.)}(\Omega)$, with $\frac{1}{p_{i}(x)}+\frac{1}{p_{i}^{\prime}(x)}=1$.

- $\left(H_{3}\right)$ : there exists a positive constant $C_{2}$ such that

$$
\left(a_{i}(x, \xi)-a_{i}(x, \eta)\right) \cdot(\xi-\eta) \geq \begin{cases}C_{2}|\xi-\eta|^{p_{i}(x)} & \text { if }|\xi-\eta| \geq 1, \\ C_{2}|\xi-\eta|^{p_{i}^{-}} & \text {if }|\xi-\eta|<1,\end{cases}
$$

for almost every $x \in \Omega$ and for every $\xi, \eta \in \mathbb{R}^{N}$, with $\xi \neq \eta$.

- $\left(H_{4}\right)$ : For almost every $x \in \Omega$ and for every $\xi \in \mathbb{R}^{N}$,

$$
|\xi|^{p_{i}(x)} \leq a_{i}(x, \xi) \cdot \xi \leq p_{i}(x) A_{i}(x, \xi) .
$$

- $\left(H_{5}\right)$ : We also assume that the variable exponents $p_{i}():. \bar{\Omega} \rightarrow[2, N)$ are continuous functions for all $i=1, \ldots, N$ such that

$$
\frac{\bar{p}(N-1)}{N(\bar{p}-1)}<p_{i}^{-}<\frac{\bar{p}(N-1)}{N-\bar{p}}, \sum_{i=1}^{N} \frac{1}{p_{i}^{-}}>1 \text { and } \frac{p_{i}^{+}-p_{i}^{-}-1}{p_{i}^{-}}<\frac{\bar{p}-N}{\bar{p}(N-1)},
$$

where $\frac{1}{\bar{p}}=\frac{1}{N} \sum_{i=1}^{N} \frac{1}{p_{i}^{-}}$.

We put for all $x \in \partial \Omega$,

$$
p^{\partial}(x)= \begin{cases}\frac{(N-1) p(x)}{N-p(x)} & \text { if } p(x)<N \\ \infty & \text { if } p(x) \geq N\end{cases}
$$

We introduce the numbers

$$
q=\frac{N(\bar{p}-1)}{N-1}, q^{*}=\frac{N q}{N-q}=\frac{N(\bar{p}-1)}{N-\bar{p}} .
$$

Non-local boundary value problems of various kinds for partial differential equations are of great interest by now in several fields of application. In a typical non-local problem, the partial differential equation (resp. boundary conditions) for an unknown function $u$ at any point in a domain $\Omega$ involves not only the local behavior of $u$ in a neighborhood of that point but also the non-local behavior of $u$ elsewhere in $\Omega$. For example, at any point in $\Omega$ the partial differential equation and/or the boundary conditions may contains integrals of the unknown $u$ over parts of $\Omega$, values of $u$ elsewhere in $D$ or, generally speaking, some non-local operator on $u$. Beside the 
mathematical interest of nonlocal conditions, it seems that this type of boundary condition appears in petroleum engineering model for well modeling in a $3 D$ stratified petroleum reservoir with arbitrary geometry (see [5] and [6]). All papers on problems like (1.1) considered cases of generally boundary value condition. Indeed, in [8], Bonzi et al. studied the following problems:

$$
\begin{cases}-\sum_{i=1}^{N} \frac{\partial}{\partial x_{i}} a_{i}\left(x, \frac{\partial}{\partial x_{i}} u\right)+|u|^{P_{M}(x)-2} u=f & \text { in } \Omega \\ \left.\sum_{i=1}^{N} a_{i}\left(x, \frac{\partial}{\partial x_{i}} u\right) \eta_{i}\right)=-|u|^{r(x)-2} u & \text { on } \partial \Omega,\end{cases}
$$

which correspond to the Robin type boundary condition. The authors used minimization techniques used in [4] to prove the existence and uniqueness of entropy solution. By the same techniques, Koné and al. proved the existence and uniqueness of entropy solution for the following problem:

$$
\begin{cases}-\sum_{i=1}^{N} \frac{\partial}{\partial x_{i}} a_{i}\left(x, \frac{\partial}{\partial x_{i}} u\right)+|u|^{P_{M}(x)-2} u=f & \text { in } \Omega \\ \left.\sum_{i=1}^{N} a_{i}\left(x, \frac{\partial}{\partial x_{i}} u\right) \eta_{i}\right)+\lambda u=g & \text { on } \partial \Omega,\end{cases}
$$

which correspond to the Fourier type boundary condition.

\section{Preliminary}

This part is related to anisotropic Lebesgue and Sobolev spaces with variable exponent and some of their properties.

Given a measurable function $p():. \Omega \rightarrow[1, \infty)$. We define the Lebesgue space with variable exponent $L^{p(.)}(\Omega)$ as the set of all measurable functions $u: \Omega \rightarrow \mathbb{R}$ for which the convex modular

$$
\rho_{p(.)}(u):=\int_{\Omega}|u|^{p(x)} d x
$$

is finite.

If the exponent is bounded, i.e., if $p_{+}<\infty$, then the expression

$$
|u|_{p(.)}:=\inf \left\{\lambda>0: \rho_{p(.)}\left(\frac{u}{\lambda}\right) \leq 1\right\}
$$

defines a norm in $L^{p(.)}(\Omega)$, called the Luxembourg norm. The space $\left(L^{p(\cdot)}(\Omega),|\cdot|_{p(.)}\right)$ is a separable Banach space. Then, $L^{p(.)}(\Omega)$ is uniformly convex, hence reflexive and its dual space is isomorphic to $L^{p^{\prime}(.)}(\Omega)$, where $\frac{1}{p(x)}+\frac{1}{p^{\prime}(x)}=1$, for all $x \in \Omega$.

Finally, we have the Hölder type inequality.

Proposition 2.1. (see [9])

(i) For any $u \in L^{p(.)}(\Omega)$ and $v \in L^{p^{\prime}(.)}(\Omega)$, we have

$$
\left|\int_{\Omega} u v d x\right| \leq\left(\frac{1}{p^{-}}+\frac{1}{p^{\prime-}}\right)|u|_{p(.)}|v|_{p^{\prime}(.)} .
$$

(ii) If $p_{1}, p_{2} \in C_{+}(\bar{\Omega}), p_{1}(x) \leq p_{2}(x)$ for any $x \in \bar{\Omega}$, then $L^{p_{2}(.)}(\Omega) \hookrightarrow L^{p_{1}(.)}(\Omega)$ and the imbedding is continuous. 
We have the following properties (see [9]) on the modular $\rho_{p(.)}$.

If $u \in L^{p(\cdot)}(\Omega)$ and $p<\infty$, then

$$
\begin{gathered}
|u|_{p(.)}<1 \Rightarrow|u|_{p(.)}^{p^{+}} \leq \rho_{p(.)}(u) \leq|u|_{p(.)}^{p^{-}}, \\
|u|_{p(.)}>1 \Rightarrow|u|_{p(.)}^{p^{-}} \leq \rho_{p(.)}(u) \leq|u|_{p(.)}^{p^{+}}, \\
|u|_{p(.)}<1(=1 ;>1) \Rightarrow \rho_{p(.)}(u)<1(=1 ;>1),
\end{gathered}
$$

and

$$
|u|_{p(.)} \rightarrow 0\left(|u|_{p(.)} \rightarrow \infty\right) \Leftrightarrow \rho_{p(.)}(u) \rightarrow 0\left(\rho_{p(.)}(u) \rightarrow \infty\right) .
$$

If in addition, $\left(u_{n}\right)_{n \in \mathbb{N}} \subset L^{p(.)}(\Omega)$, then $\lim _{n \rightarrow \infty}\left|u_{n}-u\right|_{p(.)}=0 \Leftrightarrow \lim _{n \rightarrow \infty} \rho_{p(.)}\left(u_{n}-u\right)=0 \Leftrightarrow\left(u_{n}\right)_{n \in \mathbb{N}}$ converges to $u$ in measure and $\lim _{n \rightarrow \infty} \rho_{p(.)}\left(u_{n}\right)=\rho_{p(.)}(u)$.

We introduce the definition of the isotropic Sobolev space with variable exponent,

$$
W^{1, p(.)}(\Omega):=\left\{u \in L^{p(\cdot)}(\Omega):|\nabla u| \in L^{p(\cdot)}(\Omega)\right\}
$$

which is a Banach space equipped with the norm

$$
\|u\|_{1, p(.)}:=|u|_{p(.)}+|\nabla u|_{p(.)} .
$$

Now, we present the anisotropic Sobolev space with variable exponent which is used for the study of $P(\rho, d, f)$. The anisotropic variable exponent Sobolev space $W^{1, \vec{p}(.)}(\Omega)$ is defined as follow.

$$
W^{1, \vec{p}(\cdot)}(\Omega):=\left\{u \in L^{p_{M}(\cdot)}(\Omega): \frac{\partial u}{\partial x_{i}} \in L^{p_{i}(\cdot)}(\Omega), \text { for all } i \in\{1, \ldots, N\}\right\} .
$$

Endowed with the norm

$$
\|u\|_{\vec{p}(.)}:=|u|_{p_{M}(.)}+\sum_{i=1}^{N}\left|\frac{\partial u}{\partial x_{i}}\right|_{p_{i}(.)},
$$

the space $\left(W^{1, \vec{p}(.)}(\Omega),\|\cdot\|_{\vec{p}(.)}\right)$ is a reflexive Banach space (see [10], Theorem 2.1 and Theorem 2.2). As consequence, we have the following.

Theorem 2.1. (see [10]) Let $\Omega \subset \mathbb{R}^{\mathbb{N}}(N \geq 3)$ be a bounded open set and for all $i \in\{1, \ldots, N\}, p_{i} \in L^{\infty}(\Omega), p_{i}(x) \geq 1$ a.e. in $\Omega$. Then, for any $r \in L^{\infty}(\Omega)$ with $r(x) \geq 1$ a.e. in $\Omega$ such that

$$
\text { ess } \inf _{x \in \Omega}\left(p_{M}(x)-r(x)\right)>0
$$

we have the compact embedding

$$
W^{1, \vec{p}(\cdot)}(\Omega) \hookrightarrow L^{r(\cdot)}(\Omega)
$$

We also need the following trace theorem due to [4]. 
Theorem 2.2. Let $\Omega \subset \mathbb{R}^{\mathbb{N}}(N \geq 2)$ be a bounded open set with smooth boundary and let $\vec{p}(.) \in C(\bar{\Omega})$ satisfy the condition

$$
1 \leq r(x)<\min _{x \in \partial \Omega}\left\{p_{1}^{\partial}(x), \ldots, p_{N}^{\partial}(x)\right\}, \forall x \in \partial \Omega
$$

Then, there is a compact boundary trace embedding

$$
W^{1, \vec{p}(\cdot)}(\Omega) \hookrightarrow L^{r(\cdot)}(\partial \Omega) .
$$

Let us introduce the following notation:

$$
\vec{p}_{-}=\left(p_{1}^{-}, \ldots, p_{N}^{-}\right)
$$

Finally, in this paper, we will use the Marcinkiewicz spaces $\mathcal{M}^{q}(\Omega)(1<q<\infty)$ with constant exponent. Note that the Marcinkiewicz spaces $\mathcal{M}^{q(.)}(\Omega)$ in the variable exponent setting was introduced for the first time by Sanchon and Urbano (see [11]).

Marcinkiewicz spaces $\mathcal{M}^{q}(\Omega)(1<q<\infty)$ contain all measurable function $h: \Omega \rightarrow \mathbb{R}$ for which the distribution function

$$
\lambda_{h}(\gamma):=\operatorname{meas}(\{x \in \Omega:|h(x)|>\gamma\}), \gamma \geq 0,
$$

satisfies an estimate of the form $\lambda_{h}(\gamma) \leq C \gamma^{-q}$, for some finite constant $C>0$.

The space $\mathcal{M}^{q}(\Omega)$ is a Banach space under the norm

$$
\|h\|_{\mathcal{M}^{q}(\Omega)}^{*}=\sup _{t>0} t^{\frac{1}{q}}\left(\frac{1}{t} \int_{0}^{t} h^{*}(s) d s\right)
$$

where $h^{*}$ denotes the nonincreasing rearrangement of $h$ :

$$
h^{*}(t):=\inf \left\{C: \lambda_{h}(\gamma) \leq C \gamma^{-q}, \forall \gamma>0\right\}
$$

which is equivalent to the norm $\|h\|_{\mathcal{M}^{q}(\Omega)}^{*}$ (see [1]).

We need the following Lemma (see [2], Lemma A-2).

Lemma 2.1. Let $1 \leq q<p<\infty$. Then, for every measurable function u on $\Omega$,

(i) $\frac{(p-1)^{p}}{p^{p+1}}\|u\|_{\mathcal{M}^{p}(\Omega)}^{p} \leq \sup _{\lambda>0}\left\{\lambda^{p}\right.$ meas $\left.[x \in \Omega:|u|>\lambda]\right\} \leq\|u\|_{\mathcal{M}^{p}(\Omega)}^{p}$. Moreover,

(ii) $\int_{K}|u|^{q} d x \leq \frac{p}{p-q}\left(\frac{p}{q}\right)^{\frac{q}{p}}\|u\|_{\mathcal{M}^{p}(\Omega)}^{q}(\text { meas }(K))^{\frac{p-q}{p}}$, for every measurable subset $K \subset \Omega$.

In particular, $\mathcal{M}^{p}(\Omega) \subset L_{\text {loc }}^{q}(\Omega)$, with continuous embedding and $u \in \mathcal{M}^{p}(\Omega)$ implies $|u|^{q} \in \mathcal{M}^{\frac{p}{q}}(\Omega)$.

The following result is due to Troisi (see [12]).

Theorem 2.3. Let $p_{1}, \ldots, p_{N} \in[1, \infty), \vec{p}=\left(p_{1}, \ldots, p_{N}\right) ; g \in W^{1, \vec{p}}(\Omega)$, and let

$$
\begin{cases}q=\bar{p}^{*} & \text { if } \bar{p}^{*}<N, \\ q \in[1, \infty) & \text { if } \bar{p}^{*} \geq N\end{cases}
$$


where $p^{*}=\frac{N}{\sum_{i=1}^{N} \frac{1}{p_{i}}-1}, \sum_{i=1}^{N} \frac{1}{p_{i}}>1$ and $\bar{p}^{*}=\frac{N \bar{p}}{N-\bar{p}}$.

Then, there exists a constant $C>0$ depending on $N, p_{1}, \ldots, p_{N}$ if $\bar{p}<N$ and also on $q$ and meas $(\Omega)$ if $\bar{p} \geq N$ such that

$$
\|g\|_{L^{q}(\Omega)} \leq c \prod_{i=1}^{N}\left[\|g\|_{L^{p_{M}(\Omega)}}+\left\|\frac{\partial g}{\partial x_{i}}\right\|_{L^{p_{i}}(\Omega)}\right]^{\frac{1}{N}}
$$

where $p_{M}=\max \left\{p_{1}, \ldots, p_{N}\right\}$ and $\frac{1}{\bar{p}}=\frac{1}{N} \sum_{i=1}^{N} \frac{1}{p_{i}}$. In particular, if $u \in W_{0}^{1, \vec{p}}(\Omega)$, we have

$$
\|g\|_{L^{q}(\Omega)} \leq c \prod_{i=1}^{N}\left[\left\|\frac{\partial g}{\partial x_{i}}\right\|_{L^{p_{i}(\Omega)}}\right]^{\frac{1}{N}} .
$$

In the sequel, we consider the following spaces.

$$
W_{D}^{1, \vec{p}(\cdot)}(\Omega)=\left\{\xi \in W^{1, \vec{p}(\cdot)}(\Omega): \xi=0 \text { on } \Gamma_{D}\right\}
$$

and

$$
\begin{gathered}
W_{N e}^{1, \vec{p}(.)}(\Omega)=\left\{\xi \in W_{D}^{1, \vec{p}(.)}(\Omega): \xi \equiv \text { constant on } \Gamma_{N e}\right\} . \\
\mathcal{T}_{D}^{1, \vec{p}(.)}(\Omega)=\left\{\xi \text { measurable on } \Omega \text { such that } \forall k>0, T_{k}(\xi) \in W_{D}^{1, \vec{p}(.)}(\Omega)\right\}
\end{gathered}
$$

and

$$
\mathcal{T}_{N e}^{1, \vec{p}(\cdot)}(\Omega)=\left\{\xi \text { measurable on } \Omega \text { such that } \forall k>0, T_{k}(\xi) \in W_{N e}^{1, \vec{p}(\cdot)}(\Omega)\right\}
$$

where

$$
T_{k}(s)= \begin{cases}k & \text { if } s>k \\ s & \text { if }|s| \leq k \\ -k & \text { if } s<-k\end{cases}
$$

For any $v \in W_{N e}^{1, \vec{p}(.)}(\Omega)$, we set $v_{N}=v_{N e}:=\left.v\right|_{\Gamma_{N e}}$.

Definition 2.1. A measurable function $u: \Omega \rightarrow \mathbb{R}$ is an entropy solution of $P(\rho, f, d)$ if $u \in \mathcal{T}_{N e}^{1, \vec{p}(.)}(\Omega)$ and

$$
\left\{\begin{array}{l}
\int_{\Omega}\left(\sum_{i=1}^{N} a_{i}\left(x, \frac{\partial}{\partial x_{i}} u\right) \frac{\partial}{\partial x_{i}} T_{k}(u-\xi)\right) d x+\int_{\Omega}|u|^{P_{M}(x)-2} u T_{k}(u-\xi) d x \leq \\
\int_{\Omega} f T_{k}(u-\xi) d x+\left(d-\rho\left(u_{N e}\right)\right) T_{k}\left(u_{N e}-\xi\right),
\end{array}\right.
$$

for all $\xi \in W_{N e}^{1, \vec{p}(.)}(\Omega) \cap L^{\infty}(\Omega)$.

Our main result in this paper is the following theorem.

Theorem 2.4. For any $(f, d) \in L^{1}(\Omega) \times \mathbb{R}$, the problem $P(\rho, f, d)$ admits a unique entropy solution $u$.

Before proving Theorem 2.4, we study an auxiliary problem from which, we deduce useful a priori estimates. The paper is organized as follows. In Section 3, we study an approximated problem and in Section 4, we prove by using the results of the Section 3, the existence and uniqueness of entropy solution of problem $P(\rho, f, d)$. 
3. The APPRoximAted PROBlem CORRESPONDING to $P(\rho, f, d)$

We define a new bounded domain $\tilde{\Omega}$ in $\mathbb{R}^{N}$ as follow.

We fix $\theta>0$ and we set $\tilde{\Omega}=\Omega \cup\left\{x \in \mathbb{R}^{N} / \operatorname{dist}\left(x, \Gamma_{N e}\right)<\theta\right\}$. Then, $\partial \tilde{\Omega}=\Gamma_{D} \cup \tilde{\Gamma}_{N e}$ is Lipschitz with $\Gamma_{D} \cap \tilde{\Gamma}_{N e}=\emptyset$.

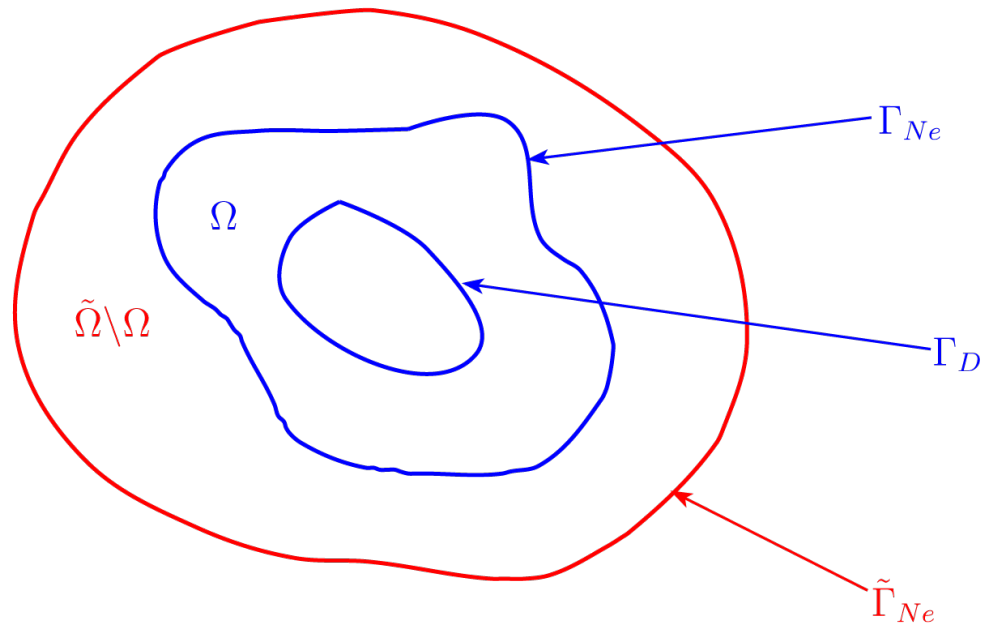

FIGURE 1. Figure 1: Domains representation

Let us consider $\tilde{a}_{i}(x, \xi)$ (to be defined later) Carathéodory and satisfying (1.4), (1.5), (1.6) and (1.7), for all $x \in \tilde{\Omega}$.

We also consider a function $\tilde{d}$ in $L^{1}\left(\tilde{\Gamma}_{N e}\right)$ such that

$$
\int_{\tilde{\Gamma}_{N e}} \tilde{d} d \sigma=d
$$

For any $\epsilon>0$, we set $f_{\epsilon}=T_{\frac{1}{\epsilon}}(f)$ and $\tilde{f}_{\epsilon}=f_{\epsilon} \chi_{\Omega}(x), \tilde{d}_{\epsilon}=T_{\frac{1}{\epsilon}}(\tilde{d})$ and we consider the problem

$$
P_{\epsilon}\left(\tilde{\rho}, \tilde{f}_{\epsilon}, \tilde{d}_{\epsilon}\right) \begin{cases}-\sum_{i=1}^{N} \frac{\partial}{\partial x_{i}} \tilde{a}_{i}\left(x, \frac{\partial}{\partial x_{i}} u_{\epsilon}\right)+\left|u_{\epsilon}\right|^{P_{M}(x)-2} u_{\epsilon} \chi_{\Omega}(x)=\tilde{f}_{\epsilon} & \text { in } \tilde{\Omega} \\ u_{\epsilon}=0 & \text { on } \Gamma_{D} \\ \tilde{\rho}\left(u_{\epsilon}\right)+\sum_{i=1}^{N} \tilde{a}_{i}\left(x, \frac{\partial}{\partial x_{i}} u_{\epsilon}\right) \eta_{i}=\tilde{d}_{\epsilon} & \text { on } \tilde{\Gamma}_{N e},\end{cases}
$$

where the function $\tilde{\rho}$ is defined as follow.

$$
\text { - } \tilde{\rho}(s)=\frac{1}{\left|\tilde{\Gamma}_{N e}\right|} \rho(s) \text {, where }\left|\tilde{\Gamma}_{N e}\right| \text { denotes the Hausdorff measure of } \tilde{\Gamma}_{N e} \text {. }
$$

We obviously have $\forall \epsilon>0, \tilde{f}_{\epsilon} \in L^{\infty}(\tilde{\Omega}), \tilde{d}_{\epsilon} \in L^{\infty}\left(\tilde{\Gamma}_{N e}\right)$.

The following definition gives the notion of solution for the problem $P_{\epsilon}\left(\tilde{\rho}, \tilde{f}_{\epsilon}, \tilde{d}_{\epsilon}\right)$.

Definition 3.1. A measurable function $u_{\epsilon}: \tilde{\Omega} \rightarrow \mathbb{R}$ is a solution to problem $P_{\epsilon}\left(\tilde{\rho}, \tilde{f}_{\epsilon}, \tilde{d}_{\epsilon}\right)$ if $u_{\epsilon} \in W_{D}^{1, \vec{p}(.)}(\tilde{\Omega})$ and

$$
\int_{\tilde{\Omega}} \sum_{i=1}^{N} \tilde{a}_{i}\left(x, \frac{\partial}{\partial x_{i}} u_{\epsilon}\right) \frac{\partial}{\partial x_{i}} \tilde{\xi} d x+\int_{\Omega}\left|u_{\epsilon}\right|^{P_{M}(x)-2} u_{\epsilon} \tilde{\xi} d x=\int_{\Omega} f_{\epsilon} \tilde{\xi} d x+\int_{\tilde{\Gamma}_{N e}}\left(\tilde{d}_{\epsilon}-\tilde{\rho}\left(u_{\epsilon}\right)\right) \tilde{\xi} d \sigma,
$$

for any $\tilde{\xi} \in W_{D}^{1, \vec{p}(.)}(\tilde{\Omega}) \cap L^{\infty}(\Omega)$. 
Theorem 3.1. The problem $P_{\epsilon}\left(\tilde{\rho}, \tilde{f}_{\epsilon}, \tilde{d}_{\epsilon}\right)$ admits at least one solution in the sense of Definition 3.1.

Step 1: Approximate problem. we study an existence result to the following problem. For any $k>0$ we consider

$$
P_{\epsilon, k}\left(\tilde{\rho}, \tilde{f}_{\epsilon}, \tilde{d}_{\epsilon}\right) \begin{cases}-\sum_{i=1}^{N} \frac{\partial}{\partial x_{i}} \tilde{a}_{i}\left(x, \frac{\partial}{\partial x_{i}} u_{\epsilon, k}\right)+T_{k}\left(b\left(u_{\epsilon, k}\right)\right) \chi_{\Omega}(x)=\tilde{f}_{\epsilon} & \text { in } \tilde{\Omega} \\ u_{\epsilon, k}=0 & \text { on } \Gamma_{D} \\ T_{k}\left(\tilde{\rho}\left(u_{\epsilon, k}\right)\right)+\sum_{i=1}^{N} \tilde{a}_{i}\left(x, \frac{\partial}{\partial x_{i}} u_{\epsilon, k}\right) \eta_{i}=\tilde{d}_{\epsilon} & \text { on } \tilde{\Gamma}_{N e}\end{cases}
$$

where $b(u)=|u|^{P_{M}(x)-2} u$.

We have to prove that $P_{\epsilon, k}\left(\tilde{\rho}, \tilde{f}_{\epsilon}, \tilde{d}_{\epsilon}\right)$ admits at least one solution in the following sense.

$$
\left\{\begin{array}{l}
u_{\epsilon, k} \in W_{D}^{1, \vec{p}(.)}(\tilde{\Omega}) \text { and for all } \tilde{\xi} \in W_{D}^{1, \vec{p}(.)}(\tilde{\Omega}) \\
\int_{\tilde{\Omega}} \sum_{i=1}^{N} \tilde{a}_{i}\left(x, \frac{\partial}{\partial x_{i}} u_{\epsilon, k}\right) \frac{\partial}{\partial x_{i}} \tilde{\xi} d x+\int_{\Omega} T_{k}\left(b\left(u_{\epsilon, k}\right)\right) \tilde{\xi} d x=\int_{\Omega} f_{\epsilon} \tilde{\xi} d x+\int_{\tilde{\Gamma}_{N e}}\left(\tilde{d}_{\epsilon}-T_{k}\left(\tilde{\rho}\left(u_{\epsilon, k}\right)\right) \tilde{\xi} d \sigma .\right.
\end{array}\right.
$$

For any $k>0$, let us introduce the operator $\Lambda_{k}: W_{D}^{1, \vec{p}(.)}(\tilde{\Omega}) \rightarrow\left(W_{D}^{1, \vec{p}(.)}(\tilde{\Omega})\right)^{\prime}$ such that for any $(u, v) \in W_{D}^{1, \vec{p}(.)}(\tilde{\Omega}) \times$ $W_{D}^{1, \vec{p}(.)}(\tilde{\Omega})$,

$$
\left\langle\Lambda_{k}(u), v\right\rangle=\int_{\tilde{\Omega}}\left(\sum_{i=1}^{N} \tilde{a}_{i}\left(x, \frac{\partial}{\partial x_{i}} u\right) \frac{\partial}{\partial x_{i}} v\right) d x+\int_{\Omega} T_{k}(b(u)) v d x+\int_{\tilde{\Gamma}_{N e}} T_{k}(\tilde{\rho}(u)) v d \sigma .
$$

We need to prove that for any $k>0$, the operator $\Lambda_{k}$ is bounded, coercive, of type $M$ and therefore, surjective.

(i) Boundedness of $\Lambda_{k}$. Let $(u, v) \in F \times W_{D}^{1, \vec{p}(.)}(\tilde{\Omega})$ with $F$ a bounded subset of $W_{D}^{1, \vec{p}(.)}(\tilde{\Omega})$. We have

$$
\left\{\begin{array}{l}
\left|\left\langle\Lambda_{k}(u), v\right\rangle\right| \leq \sum_{i=1}^{N}\left(\int_{\tilde{\Omega}}\left|\tilde{a}_{i}\left(x, \frac{\partial}{\partial x_{i}} u\right)\right|\left|\frac{\partial}{\partial x_{i}} v\right| d x\right)+\int_{\tilde{\Omega}}\left|T_{k}(b(u))\right||v| d x \\
+\int_{\tilde{\Gamma}_{N e}}\left|T_{k}(\tilde{\rho}(u))\right||v| d \sigma \\
=I_{1}+I_{2}+I_{3},
\end{array}\right.
$$

where we denote by $I_{1}, I_{2}$ and $I_{3}$ the three terms on the right hand side of the first inequality.

By $\left(H_{2}\right)$ and the Hölder type inequality, we have

$$
\left\{\begin{array}{l}
I_{1} \leq C_{1} \sum_{i=1}^{N}\left(\int_{\tilde{\Omega}}\left|j_{i}(x)\right|\left|\frac{\partial}{\partial x_{i}} v\right| d x+\int_{\tilde{\Omega}}\left|\frac{\partial}{\partial x_{i}} u\right|^{p_{i}(x)-1}\left|\frac{\partial}{\partial x_{i}} v\right| d x\right) \\
\leq C_{1} \sum_{i=1}^{N}\left(\frac{1}{p_{i}^{\prime-}}+\frac{1}{p_{i}^{-}}\right)\left|j_{i}\right|_{p_{i}^{\prime}(.)}\left|\frac{\partial}{\partial x_{i}} v\right|_{p_{i}(.)}+\left.\left.\sum_{i=1}^{N}\left(\frac{1}{p_{i}^{\prime-}}+\frac{1}{p_{i}^{-}}\right)|| \frac{\partial}{\partial x_{i}} u\right|^{p_{i}(x)-1}\right|_{p_{i}^{\prime}(.)}\left|\frac{\partial}{\partial x_{i}} v\right|_{p_{i}(.)} .
\end{array}\right.
$$

As $u \in F, \forall i \in\{1, \ldots, N\}$, there exists a constant $M>0$ such that

$$
\left.\left.\sum_{i=1}^{N}|| \frac{\partial}{\partial x_{i}} u\right|^{p_{i}(x)-1}\right|_{p_{i}^{\prime}(.)}<M
$$

so

$$
\left.\left.|| \frac{\partial}{\partial x_{i}} u\right|^{p_{i}(x)-1}\right|_{p_{i}^{\prime}(.)}<M, \forall i \in\{1, \ldots, N\}
$$


Let $C_{4}=\max _{i=1, \ldots, N}\left\{\left.\left.|| \frac{\partial}{\partial x_{i}} u\right|^{p_{i}(x)-1}\right|_{p_{i}^{\prime}(.)}\right\}$.

As $j_{i} \in L^{p_{i}^{\prime}(.)}(\tilde{\Omega})$, we have

$$
I_{1} \leq C_{5}\left(C_{1}, p_{i}^{-},\left(p_{i}^{\prime}\right)^{-}, C_{3}\left(j_{i}\right)\right) \sum_{1}^{N}\left|\frac{\partial}{\partial x_{i}} v\right|_{p_{i}(.)}+C_{6}\left(C_{1}, p_{i}^{-},\left(p_{i}^{\prime}\right)^{-}, C_{4}\right) \sum_{i=1}^{N}\left|\frac{\partial}{\partial x_{i}} v\right|_{p_{i}(.)} .
$$

It is easy to see that

$$
I_{2} \leq k \int_{\tilde{\Omega}}|v| d x .
$$

Using Theorem 2.1, we have

$$
\|v\|_{L^{1}(\tilde{\Omega})} \leq C_{7}\|v\|_{W_{D}^{1, \vec{p}(.)}(\tilde{\Omega})} .
$$

So,

$$
I_{2} \leq k C_{7}\|v\|_{W_{D}^{1, \vec{p}(\cdot)}(\tilde{\Omega})} .
$$

Similarly, by using Theorem 2.2, we have

$$
I_{3} \leq k C_{8}\|v\|_{W_{D}^{1, \vec{p}(\cdot)}(\tilde{\Omega})} \square
$$

Therefore, $\Lambda_{k}$ maps bounded subsets of $W_{D}^{1, \vec{p}(.)}(\tilde{\Omega})$ into bounded subsets of $\left(W_{D}^{1, \vec{p}(.)}(\tilde{\Omega})\right)^{\prime}$. Thus, $\Lambda_{k}$ is bounded on $W_{D}^{1, \vec{p}(\cdot)}(\tilde{\Omega})$.

(ii) Coerciveness of $\Lambda_{k}$. We have to show that for any $k>0, \frac{\left\langle\Lambda_{k}(u), u\right\rangle}{\|u\|_{W_{D}^{1, \vec{p}(\cdot)}(\tilde{\Omega})}} \rightarrow \infty$ as $\|u\|_{W_{D}^{1, \vec{p}(\cdot)}(\tilde{\Omega})} \rightarrow \infty$.

For any $u \in W_{D}^{1, \vec{p}(.)}(\tilde{\Omega})$, we have

$$
\left\langle\Lambda_{k}(u), u\right\rangle=\langle\Lambda(u), u\rangle+\int_{\Omega} T_{k}(b(u)) u d x+\int_{\tilde{\Gamma}_{N e}} T_{k}(\tilde{\rho}(u)) u d \sigma,
$$

where $\langle\Lambda(u), u\rangle=\sum_{i=1}^{N}\left(\int_{\tilde{\Omega}} \tilde{a}_{i}\left(x, \frac{\partial}{\partial x_{i}} u\right) \frac{\partial}{\partial x_{i}} u d x\right)$.

The last two terms on the right-hand side of (3.7) are non-negative by the monotonicity of $T_{k}, b$ and $\tilde{\rho}$.

We can assert that

$$
\left\{\begin{array}{l}
\left\langle\Lambda_{k}(u), u\right\rangle \geq\langle\Lambda(u), u\rangle \\
\geq \frac{1}{N^{p_{m}^{-}-1}}\|u\|_{W_{D}^{1, \vec{p}(\cdot)}(\tilde{\Omega})}^{p^{-}}-N
\end{array}\right.
$$

Indeed, since $\int_{\tilde{\Omega}}\left|T_{k}(b(u))\right||u| d x+\int_{\tilde{\Gamma}_{N e}}\left|T_{k}(\tilde{\rho}(u))\right||u| d \sigma \geq 0$, for all $u \in W_{D}^{1, \vec{p}(.)}(\tilde{\Omega})$, we have

$$
\left\langle\Lambda_{k}(u), u\right\rangle \geq\langle\Lambda(u), u\rangle
$$

So,

$$
\begin{aligned}
\left\langle\Lambda_{k}(u), u\right\rangle & \geq \sum_{i=1}^{N}\left(\int_{\tilde{\Omega}} \tilde{a}_{i}\left(x, \frac{\partial}{\partial x_{i}} u\right) \frac{\partial}{\partial x_{i}} u d x\right) \\
& \geq \sum_{i=1}^{N}\left(\int_{\tilde{\Omega}}\left|\frac{\partial}{\partial x_{i}} u\right|^{p_{i}(x)} d x\right) .
\end{aligned}
$$


We make the following notations:

$\mathcal{I}=\left\{i \in\{1, \ldots, N\}:\left|\frac{\partial}{\partial x_{i}} u\right|_{p_{i}(.)} \leq 1\right\}$ and $\mathcal{J}=\left\{i \in\{1, \ldots, N\}:\left|\frac{\partial}{\partial x_{i}} u\right|_{p_{i}(.)}>1\right\}$.

We have

$$
\begin{aligned}
\left\langle\Lambda_{k}(u), u\right\rangle & \geq \sum_{i \in \mathcal{I}}\left(\int_{\tilde{\Omega}}\left|\frac{\partial}{\partial x_{i}} u\right|^{p_{i}(x)} d x\right)+\sum_{i \in \mathcal{J}}\left(\int_{\tilde{\Omega}}\left|\frac{\partial}{\partial x_{i}} u\right|^{p_{i}(x)} d x\right) \\
& \geq \sum_{i \in \mathcal{I}}\left(\left|\frac{\partial}{\partial x_{i}} u\right|_{p_{i}(.)}^{p_{i}^{+}}\right)+\sum_{i \in \mathcal{J}}\left(\left|\frac{\partial}{\partial x_{i}} u\right|_{p_{i}(.)}^{p_{i}^{-}}\right) \\
& \geq \sum_{i \in \mathcal{J}}\left(\left|\frac{\partial}{\partial x_{i}} u\right|_{p_{i}(.)}^{p_{i}^{-}}\right) \\
& \geq \sum_{i \in \mathcal{J}}\left(\left|\frac{\partial}{\partial x_{i}} u\right|_{p_{i}(.)}^{p_{m}^{-}}\right) \\
& \geq \sum_{i=1}^{N}\left(\left|\frac{\partial}{\partial x_{i}} u\right|_{p_{i}(.)}^{p_{m}^{-}}\right)-\sum_{i \in \mathcal{I}}\left(\left|\frac{\partial}{\partial x_{i}} u\right|_{p_{i}(.)}^{p_{m}^{-}}\right) \\
& \geq \sum_{i=1}^{N}\left(\left|\frac{\partial}{\partial x_{i}} u\right|_{p_{i}(.)}^{p_{m}^{-}}\right)-N .
\end{aligned}
$$

We now use Jensen's inequality on the convex function $Z: \mathbb{R}^{+} \rightarrow \mathbb{R}^{+}, Z(t)=t^{p_{m}^{-}}, p_{m}^{-}>1$ to get

$$
\left\{\begin{array}{l}
\left\langle\Lambda_{k}(u), u\right\rangle \geq\langle\Lambda(u), u\rangle \\
\geq \frac{1}{N^{p_{m}^{-}-1}}\|u\|_{W_{D}^{1, \vec{p}(.)}(\tilde{\Omega})}^{p^{-}}-N
\end{array}\right.
$$

Hence, $\Lambda_{k}$ is coercive (as $\left.p_{m}^{-}>1\right)$.

(iii) The operator $\Lambda_{k}$ is of type $M$.

Lemma 3.1. ( $C f[13])$ Let $\mathcal{A}$ and $\mathcal{B}$ be two operators. If $\mathcal{A}$ is of type $M$ and $\mathcal{B}$ is monotone and weakly continuous, then $\mathcal{A}+\mathcal{B}$ is of type $M$.

Now , we set $\langle\mathcal{A} u, v\rangle:=\langle\Lambda(u), v\rangle$ and $\left\langle\mathcal{B}_{k} u, v\right\rangle:=\int_{\Omega} T_{k}(b(u)) v d x+\int_{\tilde{\Gamma}_{N e}} T_{k}(\tilde{\rho}(u)) v d \sigma$.

Then, for every $k>0$, we have $\Lambda_{k}=\mathcal{A}+\mathcal{B}_{k}$. We now have to show that for every $k>0, \mathcal{B}_{k}$ is monotone and weakly continuous, because it is well-known that $\mathcal{A}$ is of type $M$. For the monotonicity of $\mathcal{B}_{k}$, we have to show that

$$
\left\langle\mathcal{B}_{k} u-\mathcal{B}_{k} v, u-v\right\rangle \geq 0 \text { for all }(u, v) \in W_{D}^{1, \vec{p}(.)}(\tilde{\Omega}) \times W_{D}^{1, \vec{p}(.)}(\tilde{\Omega}) .
$$

We have

$$
\begin{aligned}
\left\langle\mathcal{B}_{k} u-\mathcal{B}_{k} v, u-v\right\rangle & =\int_{\Omega}\left(T_{k}(b(u))-T_{k}(b(v))\right)(u-v) d x \\
& +\int_{\tilde{\Gamma}_{N e}}\left(T_{k}(\tilde{\rho}(u))-T_{k}(\tilde{\rho}(v))\right)(u-v) d \sigma .
\end{aligned}
$$

From the monotonicity of $b, \tilde{\rho}$ and the map $T_{k}$, we conclude that

$$
\left\langle\mathcal{B}_{k} u-\mathcal{B}_{k} v, u-v\right\rangle \geq 0
$$


We need now to prove that for each $k>0$ the operator $\mathcal{B}_{k}$ is weakly continuous, that is, for all sequences $\left(u_{n}\right)_{n \in \mathbb{N}} \subset W_{D}^{1, \vec{p}(\cdot)}(\tilde{\Omega})$ such that $u_{n} \rightarrow u$ in $W_{D}^{1, \vec{p}(\cdot)}(\tilde{\Omega})$, we have $\mathcal{B}_{k} u_{n} \rightarrow \mathcal{B}_{k} u$ as $n \rightarrow \infty$.

For all $\phi \in W_{D}^{1, \vec{p}(.)}(\tilde{\Omega})$, we have

$$
\left\langle\mathcal{B}_{k} u_{n}, \phi\right\rangle:=\int_{\Omega} T_{k}\left(b\left(u_{n}\right)\right) \phi d x+\int_{\tilde{\Gamma}_{N e}} T_{k}\left(\tilde{\rho}\left(u_{n}\right)\right) \phi d \sigma .
$$

Passing to the limit in (3.9) as $n$ goes to $\infty$ and using the Lebesgue dominated convergence theorem, since $u_{n} \rightarrow u$ in $W_{D}^{1, \vec{p}(.)}(\tilde{\Omega})$; up to a subsequence, we have $u_{n} \rightarrow u$ in $L^{1}(\tilde{\Omega})$ and a.e. on $\tilde{\Omega}$. As $\left|T_{k}\left(b\left(u_{n}\right)\right) \phi\right| \leq k|\phi|$ and $\phi \in$ $W_{D}^{1, \vec{p}(.)}(\tilde{\Omega}) \hookrightarrow L^{1}(\tilde{\Omega})$, for the first term on the right-hand side of (3.9), we obtain

$$
\lim _{n \rightarrow \infty} \int_{\Omega} T_{k}\left(b\left(u_{n}\right)\right) \phi d x=\int_{\Omega} T_{k}(b(u)) \phi d x .
$$

Furthermore, since $u_{n} \rightarrow u$ in $W_{D}^{1, \vec{p}(.)}(\tilde{\Omega})$; up to a subsequence, we have $u_{n} \rightarrow u$ in $L^{1}(\partial \tilde{\Omega})$ and a.e. on $\partial \tilde{\Omega}$. As $\left|T_{k}\left(\tilde{\rho}\left(u_{n}\right)\right) \phi\right| \leq k|\phi|$ and $\phi \in W_{D}^{1, \vec{p}(.)}(\tilde{\Omega}) \hookrightarrow L^{1}(\partial \tilde{\Omega})$, we deduce by the Lebesgue dominated convergence theorem that

$$
\lim _{n \rightarrow \infty} \int_{\tilde{\Gamma}_{N e}} T_{k}\left(\tilde{\rho}\left(u_{n}\right)\right) \phi d x=\int_{\tilde{\Gamma}_{N e}} T_{k}(\tilde{\rho}(u)) \phi d x .
$$

From (3.10) and (3.11) we conclude that for every $k>0, \mathcal{B}_{k}\left(u_{n}\right) \rightarrow \mathcal{B}_{k}(u)$ as $n \rightarrow \infty$.

The operator $\mathcal{A}$ is type $M$ and as $\mathcal{B}_{k}$ is monotone and weakly continuous, thanks to Lemma 3.1 , we conclude that the operator $\Lambda_{k}$ is of type $M$. Then for any $L \in\left(W_{D}^{1, \vec{p}(.)}(\tilde{\Omega})\right)^{\prime}$, there exists $u_{\epsilon, k} \in W_{D}^{1, \vec{p}(.)}(\tilde{\Omega})$, such that $\Lambda_{k}\left(u_{\epsilon, k}\right)=L$.

We now consider $L \in\left(W_{D}^{1, \vec{p}(.)}(\tilde{\Omega})\right)^{\prime}$ defined by $L(v)=\int_{\Omega} f_{\epsilon} v d x+\int_{\tilde{\Gamma}_{N e}} \tilde{d}_{\epsilon} v d \sigma$, for $v \in W_{D}^{1, \vec{p}(.)}(\tilde{\Omega})$ and we obtain

Step 2: A priori estimates.

Lemma 3.2. Let $u_{\epsilon, k}$ a solution of $P_{\epsilon, k}\left(\tilde{\rho}, \tilde{f}_{\epsilon}, \tilde{d}_{\epsilon}\right)$. Then

$$
\left\{\begin{array}{l}
\left|\tilde{\rho}\left(u_{\epsilon, k}\right)\right| \leq k_{1}:=\max \left\{\|\tilde{d}\|_{\infty},\left(\tilde{\rho} \circ b^{-1}\right)\left(\left\|f_{\epsilon}\right\|_{\infty}\right)\right\} \text { a.e. on } \tilde{\Gamma}_{N e}, \\
\left|b\left(u_{\epsilon, k}\right)\right| \leq k_{2}:=\max \left\{\mid f_{\epsilon} \|_{\infty} ;\left(b \circ \rho_{0}^{-1}\right)\left(\left|\tilde{\Gamma}_{N e}\right|\|\tilde{d}\|_{\infty}\right)\right\} \text { a.e. in } \Omega .
\end{array}\right.
$$

Proof. For any $\tau>0$, let us introduce the function $H_{\tau}: \mathbb{R} \rightarrow \mathbb{R}$ by

$$
H_{\tau}(s)= \begin{cases}0 & \text { if } s<0 \\ \frac{s}{\tau} & \text { if } 0 \leq s \leq \tau \\ 1 & \text { if } s>\tau\end{cases}
$$

In (3.5) we set $\tilde{\xi}=H_{\tau}\left(u_{\epsilon, k}-M\right)$, where $M>0$ is to be fixed later. We get

$$
\left\{\begin{array}{l}
\int_{\tilde{\Omega}} \sum_{i=1}^{N} \tilde{a}_{i}\left(x, \frac{\partial}{\partial x_{i}} u_{\epsilon, k}\right) \frac{\partial}{\partial x_{i}} H_{\tau}\left(u_{\epsilon, k}-M\right) d x+\int_{\Omega} T_{k}\left(b\left(u_{\epsilon, k}\right)\right) H_{\tau}\left(u_{\epsilon, k}-M\right) d x= \\
\int_{\Omega} f_{\epsilon} H_{\tau}\left(u_{\epsilon, k}-M\right) d x+\int_{\tilde{\Gamma}_{N e}}\left(\tilde{d}-T_{k}\left(\tilde{\rho}\left(u_{\epsilon}, k\right)\right)\right) H_{\tau}\left(u_{\epsilon, k}-M\right) d \sigma .
\end{array}\right.
$$

The first term in (3.13) is non-negative. Indeed,

$$
\int_{\tilde{\Omega}} \sum_{i=1}^{N} \tilde{a}_{i}\left(x, \frac{\partial}{\partial x_{i}} u_{\epsilon, k}\right) \frac{\partial}{\partial x_{i}} H_{\tau}\left(u_{\epsilon, k}-M\right) d x=\frac{1}{\tau} \int_{\left\{0 \leq u_{\epsilon, k}-M \leq \tau\right\}} \sum_{i=1}^{N} \tilde{a}_{i}\left(x, \frac{\partial}{\partial x_{i}} u_{\epsilon, k}\right) \frac{\partial}{\partial x_{i}} u_{\epsilon, k} d x \geq 0 .
$$


From (3.13) we obtain

$$
\int_{\Omega} T_{k}\left(b\left(u_{\epsilon, k}\right)\right) H_{\tau}\left(u_{\epsilon, k}-M\right) d x \leq \int_{\Omega} f_{\epsilon} H_{\tau}\left(u_{\epsilon, k}-M\right) d x+\int_{\tilde{\Gamma}_{N e}}\left(\tilde{d}-T_{k}\left(\tilde{\rho}\left(u_{\epsilon}, k\right)\right)\right) H_{\tau}\left(u_{\epsilon, k}-M\right) d \sigma .
$$

Then, one has

$$
\left\{\begin{array}{l}
\int_{\Omega}\left(T_{k} b\left(u_{\epsilon, k}\right)-T_{k}(b(M))\right) H_{\tau}\left(u_{\epsilon, k}-M\right) d x+ \\
\int_{\tilde{\Gamma}_{N e}}\left(T_{k}\left(\tilde{\rho}\left(u_{\epsilon}, k\right)\right)-T_{k}(\tilde{\rho}(M))\right) H_{\tau}\left(u_{\epsilon, k}-M\right) d x \leq \\
\int_{\Omega}^{N}\left(f_{\epsilon}-T_{k}(b(M))\right) H_{\tau}\left(u_{\epsilon, k}-M\right) d x+\int_{\tilde{\Gamma}_{N e}}\left(\tilde{d}-T_{k}(\tilde{\rho}(M))\right) H_{\tau}\left(u_{\epsilon, k}-M\right) d \sigma .
\end{array}\right.
$$

Letting $\tau$ goes to 0 in the above inequality, we get

$$
\left\{\begin{array}{l}
\int_{\Omega}\left(T_{k}\left(b\left(u_{\epsilon, k}\right)\right)-T_{k}(b(M))\right)^{+} d x+\int_{\tilde{\Gamma}_{N e}}\left(T_{k}\left(\tilde{\rho}\left(u_{\epsilon, k}\right)\right)-T_{k}(\tilde{\rho}(M))\right)^{+} d \sigma \leq \\
\int_{\Omega}\left(f_{\epsilon}-T_{k}(b(M))\right) \operatorname{sign}_{0}^{+}\left(u_{k}-M\right) d x+\int_{\tilde{\Gamma}_{N e}}\left(\tilde{d}-T_{k}(\tilde{\rho}(M))\right) \operatorname{sign}_{0}^{+}\left(u_{\epsilon, k}-M\right) d \sigma .
\end{array}\right.
$$

As $\operatorname{Im}(b)=\operatorname{Im}(\rho)=\mathbb{R}$, we can fix $M=M_{0}=\max \left\{b^{-1}\left(\left\|f_{\epsilon}\right\|_{\infty}\right), \rho_{0}^{-1}\left(\left|\tilde{\Gamma}_{N e}\right|\|\tilde{d}\|_{\infty}\right)\right\}$. From the above inequality we obtain

$$
\left\{\begin{array}{l}
\int_{\Omega}\left(T_{k}\left(b\left(u_{\epsilon, k}\right)\right)-T_{k}\left(b\left(M_{0}\right)\right)\right)^{+} d x+\int_{\tilde{\Gamma}_{N e}}\left(T_{k}\left(\tilde{\rho}\left(u_{\epsilon, k}\right)-T_{k}\left(\tilde{\rho}\left(M_{0}\right)\right)\right)^{+} d \sigma \leq\right. \\
\int_{\Omega}\left(f_{\epsilon}-T_{k}\left(\left\|f_{\epsilon}\right\|_{\infty}\right)\right) \operatorname{sign}_{0}^{+}\left(u_{\epsilon, k}-M_{0}\right) d x+\int_{\tilde{\Gamma}_{N e}}\left(\tilde{d}-T_{k}\left(\|\tilde{d}\|_{\infty}\right)\right) \operatorname{sign}_{0}^{+}\left(u_{\epsilon, k}-M_{0}\right) d \sigma .
\end{array}\right.
$$

For $k>k_{0}:=\max \left\{\left\|f_{\epsilon}\right\|,\|\tilde{d}\|_{\infty}\right\}$,

it follows that

$$
\int_{\Omega}\left(T_{k}\left(b\left(u_{\epsilon, k}\right)\right)-T_{k}\left(b\left(M_{0}\right)\right)\right)^{+} d x+\int_{\tilde{\Gamma}_{N e}}\left(T_{k}\left(\tilde{\rho}\left(u_{\epsilon, k}\right)\right)-T_{k}\left(\tilde{\rho}\left(M_{0}\right)\right)\right)^{+} d \sigma \leq 0 .
$$

From (3.14), we deduce that

$$
\left\{\begin{array}{l}
T_{k}\left(\tilde{\rho}\left(u_{\epsilon, k}\right)\right) \leq T_{k}\left(\tilde{\rho}\left(M_{0}\right)\right) \text { a.e. on } \tilde{\Gamma}_{N e} \\
T_{k}\left(b\left(u_{\epsilon, k}\right)\right) \leq T_{k}\left(b\left(M_{0}\right)\right) \text { a.e. in } \Omega .
\end{array}\right.
$$

From (3.15), we deduce that for every $k>k_{1}:=\max \left\{\|\tilde{d}\|_{\infty},\left\|f_{\epsilon}\right\|_{\infty}, b\left(M_{0}\right), \tilde{\rho}\left(M_{0}\right)\right\}$,

$$
\tilde{\rho}\left(u_{\epsilon, k}\right) \leq \tilde{\rho}\left(M_{0}\right) \text { a.e. on } \tilde{\Gamma}_{N e}
$$

and

$$
b\left(u_{\epsilon, k}\right) \leq b\left(M_{0}\right) \text { a.e. in } \Omega \text {. }
$$

Note that with the choice of $M_{0}$ and the fact that $D(\rho)=D(b)=\mathbb{R}$, for every $k>k_{1}:=$ $\max \left\{\|\tilde{d}\|_{\infty},\left\|f_{\epsilon}\right\|_{\infty}, b\left(M_{0}\right), \tilde{\rho}\left(M_{0}\right)\right\}$, we have

$$
\left\{\begin{array}{l}
b\left(u_{\epsilon, k}\right) \leq \max \left\{\left\|f_{\epsilon}\right\|_{\infty}, b \circ \rho_{0}^{-1}\left(\left|\tilde{\Gamma}_{N e}\right|\|\tilde{d}\|_{\infty}\right)\right\} \text { a.e. in } \Omega \\
\tilde{\rho}\left(u_{\epsilon, k}\right) \leq \max \left\{\|\tilde{d}\|_{\infty},\left(\tilde{\rho} \circ b^{-1}\right)\left(\left\|f_{\epsilon}\right\|_{\infty}\right)\right\} \text { a.e. on } \tilde{\Gamma}_{N e}
\end{array}\right.
$$


We need to show that for $k$ large enough,

$$
\left\{\begin{array}{l}
b\left(u_{\epsilon, k}\right) \geq-\max \left\{\left\|f_{\epsilon}\right\|_{\infty}, b \circ \rho_{0}^{-1}\left(\left|\tilde{\Gamma}_{N e}\right|\|\tilde{d}\|_{\infty}\right)\right\} \text { a.e. in } \Omega, \\
\tilde{\rho}\left(u_{\epsilon, k}\right) \geq-\max \left\{\|\tilde{d}\|_{\infty},\left(\tilde{\rho} \circ b^{-1}\right)\left(\left\|f_{\epsilon}\right\|_{\infty}\right)\right\} \text { a.e. on } \tilde{\Gamma}_{N e}
\end{array}\right.
$$

It is easy to see that if $u_{\epsilon, k}$ is a solution of $P_{\epsilon, k}\left(\tilde{\rho}, \tilde{f}_{\epsilon}, \tilde{d}_{\epsilon}\right)$, then $\left(-u_{\epsilon, k}\right)$ is a solution of

$$
P_{k}\left(\hat{\rho}, \hat{f}_{\epsilon}, \hat{d}_{\epsilon}\right) \begin{cases}-\sum_{i=1}^{N} \frac{\partial}{\partial x_{i}} \hat{a}_{i}\left(x, \frac{\partial}{\partial x_{i}} u_{\epsilon, k}\right)+T_{k}\left(\hat{b}\left(u_{\epsilon, k}\right)\right) \chi_{\Omega}(x)=\hat{f}_{\epsilon} & \text { in } \tilde{\Omega} \\ u_{\epsilon, k}=0 & \text { on } \Gamma_{D} \\ T_{k}\left(\hat{\rho}\left(u_{\epsilon, k}\right)\right)+\sum_{i=1}^{N} \hat{a}_{i}\left(x, \frac{\partial}{\partial x_{i}} u_{\epsilon, k}\right) \eta_{i}=\hat{d}_{\epsilon} & \text { on } \tilde{\Gamma}_{N e},\end{cases}
$$

where $\hat{a}_{i}(x, \xi)=-\tilde{a}_{i}(x,-\xi), \hat{\rho}(s)=-\tilde{\rho}(-s), \hat{b}(x, s)=-b(x,-s) \chi_{\Omega}(x), \hat{f}_{\epsilon}=-\tilde{f}_{\epsilon}$ and $\hat{d}=-\tilde{d}$.

Then, for every $k>k_{2}:=\max \left\{\|\tilde{d}\|_{\infty},\left\|f_{\epsilon}\right\|_{\infty},-b\left(-M_{0}\right),-\tilde{\rho}\left(-M_{0}\right)\right\}$, we have

$$
\left\{\begin{array}{l}
-b\left(u_{\epsilon, k}\right) \leq \max \left\{\left\|f_{\epsilon}\right\|_{\infty}, b \circ \rho_{0}^{-1}\left(\left|\tilde{\Gamma}_{N e}\right|\|\tilde{d}\|_{\infty}\right)\right\} \text { a.e. in } \Omega \\
-\tilde{\rho}\left(u_{\epsilon, k}\right) \leq \max \left\{\|\tilde{d}\|_{\infty},\left(\tilde{\rho} \circ b^{-1}\right)\left(\left\|f_{\epsilon}\right\|_{\infty}\right)\right\} \text { a.e. on } \tilde{\Gamma}_{N e}
\end{array}\right.
$$

which implies (3.17).

From (3.16) and (3.17), we deduce (3.12).

Step 3. Convergence. Since $u_{\epsilon, k}$ is a solution of $P_{\epsilon, k}\left(\tilde{\rho}, \tilde{f}_{\epsilon}, \tilde{d}_{\epsilon}\right)$, thanks to Lemma 3.2 and the fact that $\Omega$ is bounded, we have $\tilde{\rho}\left(u_{\epsilon, k}\right) \in L^{1}\left(\tilde{\Gamma}_{N e}\right)$ and $b\left(u_{\epsilon, k}\right) \in L^{1}(\Omega)$. For $k=1+\max \left(k_{1}, k_{2}\right)$ fixed, by Lemma 3.2, one sees that problem $P_{\epsilon}\left(\tilde{\rho}, \tilde{f}_{\epsilon}, \tilde{d}_{\epsilon}\right)$ admits at least one solution $u_{\epsilon}$

Remark 1. Using the relation (3.12) and the fact that the functions $b$ and $\rho$ are non-decreasing, it follows that for $k$ large enough, the solution of the problem $P\left(\tilde{\rho}, \tilde{f}_{\epsilon}, \tilde{d}_{\epsilon}\right)$ belongs to $L^{\infty}(\Omega) \cap L^{\infty}\left(\tilde{\Gamma}_{N e}\right)$ and $\left|u_{\epsilon}\right| \leq c\left(b, k_{1}\right)$ a.e. in $\Omega$ and $\left|u_{\epsilon}\right| \leq c\left(\rho, k_{2}\right)$ a.e. on $\tilde{\Gamma}_{N e}$.

Now, we set $\tilde{a}_{i}(x, \xi)=a_{i}(x, \xi) \chi_{\Omega}(x)+\frac{1}{\epsilon^{p_{i}(x)}}|\xi|^{p_{i}(x)-2} \xi \chi_{\tilde{\Omega} \backslash \Omega}(x)$ for all $(x, \xi) \in \tilde{\Omega} \times \mathbb{R}^{N}$ and we consider the following problem denoted by $P_{\epsilon}\left(\tilde{\rho}, \tilde{f}_{\epsilon}, \tilde{d}_{\epsilon}\right)$.

$$
\begin{cases}-\sum_{i=1}^{N} \frac{\partial}{\partial x_{i}}\left(a_{i}\left(x, \frac{\partial}{\partial x_{i}} u_{\epsilon}\right) \chi_{\Omega}(x)+\frac{1}{\epsilon^{p_{i}(x)}}\left|\frac{\partial}{\partial x_{i}} u_{\epsilon}\right|^{p_{i}(x)-2} \frac{\partial}{\partial x_{i}} u_{\epsilon} \chi_{\tilde{\Omega} \backslash \Omega}(x)\right)+ & \\ \left|u_{\epsilon}\right|^{P_{M}(x)-2} u_{\epsilon} \chi_{\Omega}=\tilde{f}_{\epsilon} & \text { in } \tilde{\Omega} \\ u_{\epsilon}=0 & \text { on } \Gamma_{D} \\ \tilde{\rho}\left(u_{\epsilon}\right)+\sum_{i=1}^{N} \tilde{a}_{i}\left(x, \frac{\partial}{\partial x_{i}} u_{\epsilon}\right) \eta_{i}=\tilde{d}_{\epsilon} & \text { on } \tilde{\Gamma}_{N e} .\end{cases}
$$

Thanks to Theorem 3.1, $P_{\epsilon}\left(\tilde{\rho}, \tilde{f}_{\epsilon}, \tilde{d}_{\epsilon}\right)$ has at least one solution. So, there exists at least one measurable function $u_{\epsilon}: \tilde{\Omega} \rightarrow \mathbb{R}$ such that

$$
\left\{\begin{array}{l}
\sum_{i=1}^{N} \int_{\Omega} a_{i}\left(x, \frac{\partial}{\partial x_{i}} u_{\epsilon}\right) \frac{\partial}{\partial x_{i}} \tilde{\xi} d x+\sum_{i=1}^{N} \int_{\tilde{\Omega} \backslash \Omega}\left(\frac{1}{\epsilon^{p_{i}(x)}}\left|\frac{\partial}{\partial x_{i}} u_{\epsilon}\right|^{p_{i}(x)-2} \frac{\partial}{\partial x_{i}} u_{\epsilon} \frac{\partial}{\partial x_{i}} \tilde{\xi}\right) d x \\
+\int_{\Omega}\left|u_{\epsilon}\right|^{P_{M}(x)-2} u_{\epsilon} \tilde{\xi} d x=\int_{\Omega} f_{\epsilon} \tilde{\xi} d x+\int_{\tilde{\Gamma}_{N e}}\left(\tilde{d}_{\epsilon}-\tilde{\rho}\left(u_{\epsilon}\right)\right) \tilde{\xi} d \sigma
\end{array}\right.
$$


where $u_{\epsilon} \in W_{D}^{1, \vec{p}(.)}(\tilde{\Omega})$ and $\tilde{\xi} \in W_{D}^{1, \vec{p}(.)}(\tilde{\Omega}) \cap L^{\infty}(\Omega)$.

The next result gives a priori estimates on the solution $u_{\epsilon}$ of the problem $P_{\epsilon}\left(\tilde{\rho}, \tilde{f}_{\epsilon}, \tilde{d}_{\epsilon}\right)$.

Proposition 3.1. Let $u_{\epsilon}$ be a solution of the problem $P_{\epsilon}\left(\tilde{\rho}, \tilde{f}_{\epsilon}, \tilde{d}_{\epsilon}\right)$. Then, the following statements hold.

(i) $\forall k>0$,

$$
\sum_{i=1}^{N} \int_{\Omega}\left(\frac{\partial}{\partial x_{i}}\left|T_{k}\left(u_{\epsilon}\right)\right|\right)^{p_{i}(x)} d x+\sum_{i=1}^{N} \int_{\tilde{\Omega} \backslash \Omega}\left(\frac{1}{\epsilon}\left|\frac{\partial}{\partial x_{i}} T_{k}\left(u_{\epsilon}\right)\right|\right)^{p_{i}(x)} d x \leq k\left(\|\tilde{d}\|_{L^{1}\left(\tilde{\Gamma}_{N e}\right)}+\|f\|_{L^{1}(\Omega)}\right) ;
$$

(ii)

(iii) $\forall k>0$,

$$
\int_{\Omega}\left|u_{\epsilon}\right|^{P_{M}(x)-1} d x+\int_{\tilde{\Gamma}_{N e}}\left|\tilde{\rho}\left(u_{\epsilon}\right)\right| d x \leq\left(\|\tilde{d}\|_{L^{1}\left(\tilde{\Gamma}_{N e}\right)}+\|f\|_{L^{1}(\Omega)}\right)
$$

$$
\sum_{i=1}^{N} \int_{\tilde{\Omega}}\left(\frac{\partial}{\partial x_{i}}\left|T_{k}\left(u_{\epsilon}\right)\right|\right)^{p_{i}(x)} d x \leq k\left(\|\tilde{d}\|_{L^{1}\left(\tilde{\Gamma}_{N e}\right)}+\|f\|_{L^{1}(\Omega)}\right) .
$$

Proof. For any $k>0$, we set $\tilde{\xi}=T_{k}\left(u_{\epsilon}\right)$ in (3.19), to get

$$
\left\{\begin{array}{l}
\sum_{i=1}^{N} \int_{\Omega}\left(a_{i}\left(x, \frac{\partial}{\partial x_{i}} u_{\epsilon}\right) \frac{\partial}{\partial x_{i}} T_{k}\left(u_{\epsilon}\right)\right) d x+\sum_{i=1}^{N} \int_{\tilde{\Omega} \backslash \Omega}\left(\frac{1}{\epsilon^{p_{i}(x)}}\left|\frac{\partial}{\partial x_{i}} u_{\epsilon}\right|^{p_{i}(x)-2} \frac{\partial}{\partial x_{i}} u_{\epsilon} \frac{\partial}{\partial x_{i}} T_{k}\left(u_{\epsilon}\right)\right) d x \\
\int_{\Omega}\left|u_{\epsilon}\right|^{P_{M}(x)-2} u_{\epsilon} T_{k}\left(u_{\epsilon}\right) d x=\int_{\Omega} f_{\epsilon} T_{k}\left(u_{\epsilon}\right) d x+\int_{\tilde{\Gamma}_{N e}}\left(\tilde{d}_{\epsilon}-\tilde{\rho}\left(u_{\epsilon}\right)\right) T_{k}\left(u_{\epsilon}\right) d \sigma .
\end{array}\right.
$$

(i) Obviously, we have

$\sum_{i=1}^{N} \int_{\tilde{\Omega} \backslash \Omega}\left(\frac{1}{\epsilon^{p_{i}(x)}}\left|\frac{\partial}{\partial x_{i}} u_{\epsilon}\right|^{p_{i}(x)-2} \frac{\partial}{\partial x_{i}} u_{\epsilon} \frac{\partial}{\partial x_{i}} T_{k}\left(u_{\epsilon}\right)\right) d x=\sum_{i=1}^{N} \int_{\tilde{\Omega} \backslash \Omega}\left(\frac{1}{\epsilon^{p_{i}(x)}}\left|\frac{\partial}{\partial x_{i}} T_{k}\left(u_{\epsilon}\right)\right|^{p_{i}(x)}\right) d x \quad \geq \quad 0$, $\int_{\tilde{\Gamma}_{N e}} \tilde{\rho}\left(u_{\epsilon}\right) T_{k}\left(u_{\epsilon}\right) d \sigma \geq 0$ and $\int_{\Omega}\left|u_{\epsilon}\right|^{P_{M}(x)-2} u_{\epsilon} T_{k}\left(u_{\epsilon}\right) \geq 0$.

Moreover,

$$
\left\{\begin{aligned}
\int_{\Omega} f_{\epsilon} T_{k}\left(u_{\epsilon}\right) d x+\int_{\tilde{\Gamma}_{N e}} \tilde{d}_{\epsilon} T_{k}\left(u_{\epsilon}\right) d \sigma & \leq k \int_{\Omega}\left|f_{\epsilon}\right| d x+k \int_{\tilde{\Gamma}_{N e}}\left|\tilde{d}_{\epsilon}\right| d \sigma \\
& \leq k\left(\int_{\Omega}|f| d x+\int_{\tilde{\Gamma}_{N e}}|\tilde{d}| d \sigma\right)
\end{aligned}\right.
$$

Using the inequalities above and (1.7),

it follows that

$$
\sum_{i=1}^{N} \int_{\Omega}\left|\frac{\partial T_{k}\left(u_{\epsilon}\right)}{\partial x_{i}}\right|^{p_{i}(x)} d x \leq k\left(\int_{\Omega}|f| d x+\int_{\tilde{\Gamma}_{N e}}|\tilde{d}| d \sigma\right) .
$$

In (3.20), as $\sum_{i=1}^{N} \int_{\Omega}\left(a_{i}\left(x, \frac{\partial}{\partial x_{i}} u_{\epsilon}\right) \frac{\partial}{\partial x_{i}} T_{k}\left(u_{\epsilon}\right)\right) d x \geq 0, \int_{\tilde{\Gamma}_{N e}} \tilde{\rho}\left(u_{\epsilon}\right) T_{k}\left(u_{\epsilon}\right) d \sigma \geq 0$ and $\int_{\Omega}\left|u_{\epsilon}\right|^{P_{M}(x)-2} u_{\epsilon} T_{k}\left(u_{\epsilon}\right) \geq 0$

therefore we obtain

$$
\sum_{i=1}^{N} \int_{\tilde{\Omega} \backslash \Omega}\left(\frac{1}{\epsilon^{p_{i}(x)}}\left|\frac{\partial}{\partial x_{i}} T_{k}\left(u_{\epsilon}\right)\right|^{p_{i}(x)}\right) d x \leq k\left(\int_{\Omega}|f| d x+\int_{\tilde{\Gamma}_{N e}}|\tilde{d}| d \sigma\right) .
$$

Adding (3.22) and (3.23), we obtain (i).

(ii) The two first terms in (3.20) are non-negative and using (3.21), then we have from (3.20) the following

$$
\int_{\tilde{\Gamma}_{N e}} \tilde{\rho}\left(u_{\epsilon}\right) T_{k}\left(u_{\epsilon}\right) d \sigma+\int_{\Omega}\left|u_{\epsilon}\right|^{P_{M}(x)-2} u_{\epsilon} T_{k}\left(u_{\epsilon}\right) d x \leq k\left(\int_{\Omega}|f| d x+\int_{\tilde{\Gamma}_{N e}}|\tilde{d}| d \sigma\right) .
$$


We divide the above inequality by $k>0$ and we let $k$ goes to zero, to get

$$
\begin{aligned}
\int_{\tilde{\Gamma}_{N e}} \tilde{\rho}\left(u_{\epsilon}\right) \operatorname{sign}\left(u_{\epsilon}\right) d \sigma+\int_{\Omega}\left|u_{\epsilon}\right|^{P_{M}(x)-2} u_{\epsilon} \operatorname{sign}\left(u_{\epsilon}\right) d x & =\int_{\tilde{\Gamma}_{N e}}\left|\tilde{\rho}\left(u_{\epsilon}\right)\right| d \sigma+\int_{\Omega}\left|u_{\epsilon}\right|^{P_{M}(x)-1} d x \\
& \leq\left(\int_{\Omega}|f| d x+\int_{\tilde{\Gamma}_{N e}}|\tilde{d}| d \sigma\right) .
\end{aligned}
$$

(iii) For all $k>0$, we have

$$
\left\{\begin{array}{l}
\sum_{i=1}^{N} \int_{\tilde{\Omega}}\left(\frac{\partial}{\partial x_{i}}\left|T_{k}\left(u_{\epsilon}\right)\right|^{p_{i}(x)}\right) d x \leq \sum_{i=1}^{N} \int_{\Omega}\left(\frac{\partial}{\partial x_{i}}\left|T_{k}\left(u_{\epsilon}\right)\right|^{p_{i}(x)}\right) d x \\
+\sum_{i=1}^{N} \int_{\tilde{\Omega} \backslash \Omega}\left(\frac{1}{\epsilon}\left|\frac{\partial}{\partial x_{i}} T_{k}\left(u_{\epsilon}\right)\right|\right)^{p_{i}(x)} d x
\end{array}\right.
$$

for any $0<\epsilon<1$.

According to $(i)$, we deduce that

$$
\sum_{i=1}^{N} \int_{\tilde{\Omega}}\left(\frac{\partial}{\partial x_{i}}\left|T_{k}\left(u_{\epsilon}\right)\right|^{p_{i}(x)}\right) d x \leq k\left(\|\tilde{d}\|_{L^{1}\left(\tilde{\Gamma}_{N e}\right)}+\|f\|_{L^{1}(\Omega)}\right)
$$

We have the following results.

Lemma 3.3. Assume (1.4)-(1.8), $f \in L^{1}(\Omega)$ and $\tilde{d} \in L^{1}\left(\tilde{\Gamma}_{N e}\right)$. Let $u_{\epsilon}$ be a solution of the problem $P_{\epsilon}\left(\tilde{\rho}, \tilde{f}_{\epsilon}, \tilde{d}_{\epsilon}\right)$. If there is a positive constant $M$ such that

$$
\sum_{i=1}^{N} \int_{\left\{\left|u_{\epsilon}\right|>k\right\}} k^{q_{i}(x)} d x \leq M, \text { for all } k>0
$$

then,

$$
\sum_{i=1}^{N}\left\{\left|\frac{\partial}{\partial x i} u_{\epsilon}\right|^{\alpha_{i}(.)}>k\right\} k^{k_{i}(x)} d x \leq\|\tilde{d}\|_{L^{1}\left(\tilde{\Gamma}_{N e}\right)}+\|f\|_{L^{1}(\Omega)}+M, \text { for all } k>0
$$

where $\alpha_{i}()=.\frac{p_{i}(.)}{q_{i}(.)+1}$, for all $i=1, \ldots, N$.

Proof. Having in mind that by Proposition 3.1-(iii), for all $k>0$,

$$
\sum_{i=1}^{N} \int_{\tilde{\Omega}}\left|\frac{\partial T_{k}\left(u_{\epsilon}\right)}{\partial x_{i}}\right|^{p_{i}(x)} d x \leq k\left(\int_{\Omega}|f| d x+\int_{\tilde{\Gamma}_{N e}}|\tilde{d}| d \sigma\right)
$$

Therefore, defining $\psi:=T_{k}\left(u_{\epsilon}\right) / k$, we have, for all $k>0$,

$$
\sum_{i=1}^{N} \int_{\tilde{\Omega}} k^{p_{i}(x)-1}\left|\frac{\partial \psi}{\partial x_{i}}\right|^{p_{i}(x)} d x=\sum_{i=1}^{N} \frac{1}{k} \int_{\tilde{\Omega}}\left|\frac{\partial T_{k}\left(u_{\epsilon}\right)}{\partial x_{i}}\right|^{p_{i}(x)} d x \leq\left(\int_{\Omega}|f| d x+\int_{\tilde{\Gamma}_{N e}}|\tilde{d}| d \sigma\right) .
$$


From the above inequality, the definition of $\alpha_{i}($.$) and (3.24), we have$

$$
\begin{aligned}
& \left\{\begin{array}{l}
\sum_{i=1}^{N}\left\{\left|\frac{\partial}{\partial x_{i}} u_{\epsilon}\right|^{\alpha_{i}(.)}>k\right\}^{k^{q_{i}(x)} d x \leq \sum_{i=1}^{N} \int\left\{\left|\frac{\partial}{\partial x_{i}} u_{\epsilon}\right|^{\alpha_{i}(.)}>k\right\} \cap\left\{\left|u_{\epsilon}\right| \leq k\right\}} k^{q_{i}(x)} d x \\
+\sum_{i=1}^{N} \int_{\left\{\left|u_{\epsilon}\right|>k\right\}} k^{q_{i}(x)} d x
\end{array}\right.
\end{aligned}
$$

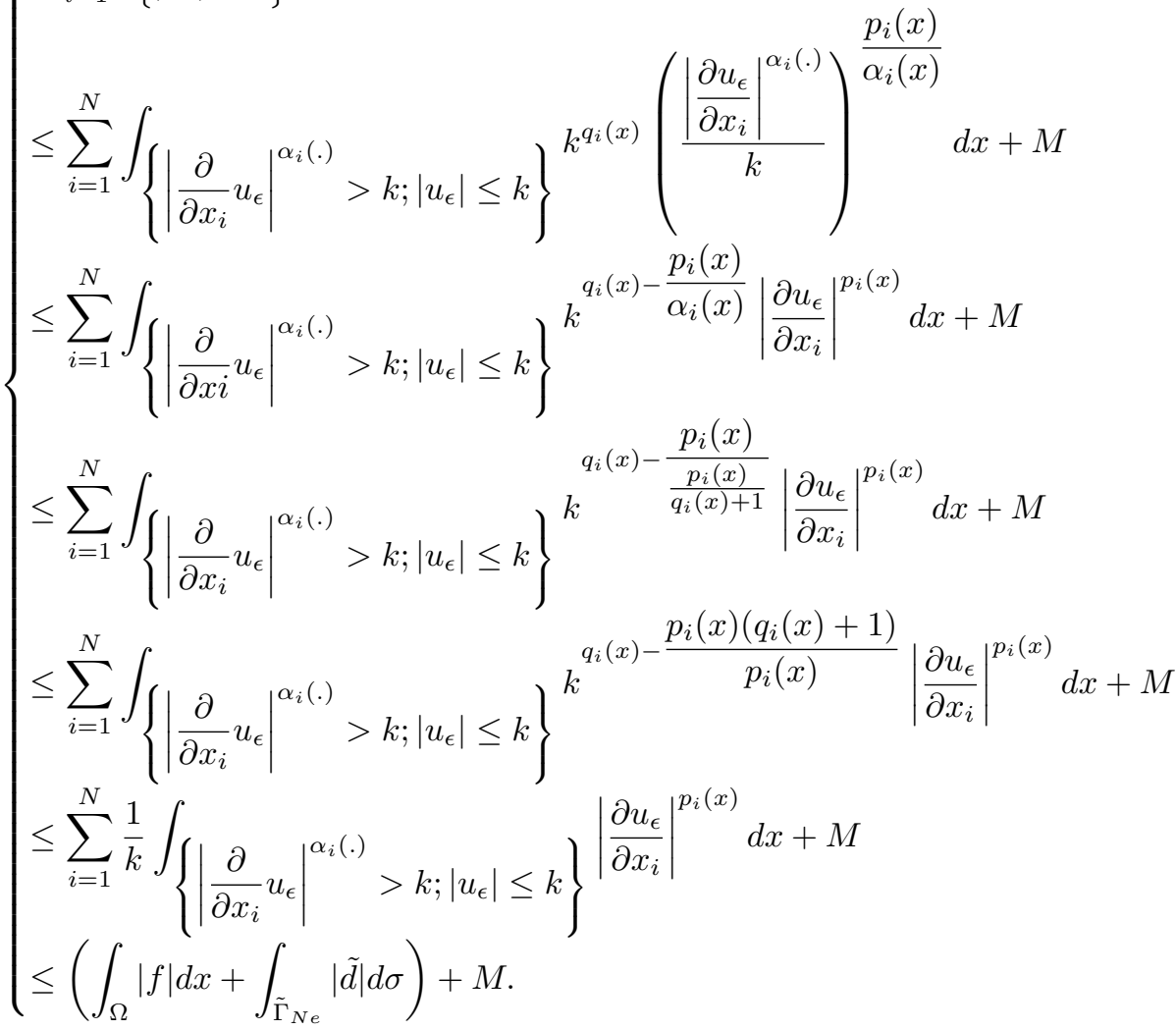

Lemma 3.4. Assume (1.4)-(1.8), $f \in L^{1}(\Omega)$ and $\tilde{d} \in L^{1}\left(\tilde{\Gamma}_{N e}\right)$. Let $u_{\epsilon}$ be a solution of the problem $P_{\epsilon}\left(\tilde{\rho}, \tilde{f}_{\epsilon}, \tilde{d}_{\epsilon}\right)$. There is a positive constant $D$ such that

$$
\operatorname{meas}\left\{\left|u_{\epsilon}\right|>k\right\} \leq D^{p_{m}^{-}} \frac{(1+k)}{k^{p_{m}^{-}-1}}, \forall k>0 .
$$

Proof. Let $k>0$; by using Proposition 3.1-(iii), we have

$$
\begin{aligned}
\sum_{i=1}^{N} \int_{\tilde{\Omega}}\left|\frac{\partial T_{k}\left(u_{\epsilon}\right)}{\partial x_{i}}\right|^{p_{m}^{-}} d x & \leq \sum_{i=1}^{N}\left\{\left|\frac{\partial T_{k}\left(u_{\epsilon}\right)}{\partial x_{i}}\right|_{>1}\right\}\left|\frac{\partial T_{k}\left(u_{\epsilon}\right)}{\partial x_{i}}\right|^{p_{m}^{-}} d x+\operatorname{Nmeas}(\tilde{\Omega}) \\
& \leq \sum_{i=1}^{N} \int_{\tilde{\Omega}}\left|\frac{\partial T_{k}\left(u_{\epsilon}\right)}{\partial x_{i}}\right|^{p_{i}(x)} d x+\operatorname{Nmeas}(\tilde{\Omega}) \\
& \leq k\left(\int_{\Omega}|f| d x+\int_{\tilde{\Gamma}_{N e}}|\tilde{d}| d \sigma\right)+\operatorname{Nmeas}(\tilde{\Omega}) \\
& \leq C^{\prime}(k+1),
\end{aligned}
$$

with $C^{\prime}=\max \left(\left(\int_{\Omega}|f| d x+\int_{\tilde{\Gamma}_{N e}}|\tilde{d}| d \sigma\right) ; \operatorname{Nmeas}(\tilde{\Omega})\right)$. 
We can write the above inequality as

$$
\sum_{i=1}^{N}\left\|\frac{\partial T_{k}\left(u_{\epsilon}\right)}{\partial x_{i}}\right\|_{p_{m}^{-}}^{p_{m}^{-}} \leq C^{\prime}(1+k) \text { or }\left\|T_{k}\left(u_{\epsilon}\right)\right\|_{W_{D}^{1, p_{m}^{-}}(\tilde{\Omega})} \leq\left[C^{\prime}(1+k)\right]^{\frac{1}{p_{m}^{-}}} .
$$

By the Poincaré inequality in constant exponent, we obtain

$$
\left\|T_{k}\left(u_{\epsilon}\right)\right\|_{L^{p^{-}}(\tilde{\Omega})} \leq D(1+k)^{\frac{1}{p_{m}^{-}}} .
$$

The above inequality implies that

$$
\int_{\tilde{\Omega}}\left|T_{k}\left(u_{\epsilon}\right)\right|^{p_{m}^{-}} d x \leq D^{p_{m}^{-}}(1+k),
$$

from which we obtain

$$
\text { meas }\left\{\left|u_{\epsilon}\right|>k\right\} \leq D^{p_{m}^{-}} \frac{(1+k)}{k^{p_{m}^{-}}},
$$

because, as

$$
\int_{\tilde{\Omega}}\left|T_{k}\left(u_{\epsilon}\right)\right|^{p_{m}^{-}} d x=\int_{\left\{\left|u_{\epsilon}\right|>k\right\}}\left|T_{k}\left(u_{\epsilon}\right)\right|^{p_{m}^{-}} d x+\int_{\left\{\left|u_{\epsilon}\right| \leq k\right\}}\left|T_{k}\left(u_{\epsilon}\right)\right|^{p_{m}^{-}} d x,
$$

we have

$$
\int_{\left\{\left|u_{\epsilon}\right|>k\right\}}\left|T_{k}\left(u_{\epsilon}\right)\right|^{p_{m}^{-}} d x \leq \int_{\tilde{\Omega}}\left|T_{k}\left(u_{\epsilon}\right)\right|^{p_{m}^{-}} d x
$$

and

$$
k^{p_{m}^{-}} \text {meas }\left\{\left|u_{\epsilon}\right|>k\right\} \leq \int_{\tilde{\Omega}}\left|T_{k}\left(u_{\epsilon}\right)\right|^{p_{m}^{-}} d x \leq D^{p_{m}^{-}}(1+k)
$$

Lemma 3.5. Assume (1.4)-(1.8), $f \in L^{1}(\Omega)$ and $\tilde{d} \in L^{1}\left(\tilde{\Gamma}_{N e}\right)$. Let $u_{\epsilon}$ be a solution of the problem $P_{\epsilon}\left(\tilde{\rho}, \tilde{f}_{\epsilon}, \tilde{d}_{\epsilon}\right)$. There is a positive constant $C$ such that

$$
\sum_{i=1}^{N} \int_{\tilde{\Omega}}\left(\left|\frac{\partial}{\partial x_{i}} T_{k}\left(u_{\epsilon}\right)\right|^{p_{i}^{-}}\right) d x \leq C(k+1), \forall k>0 .
$$

Proof. Let $k>0$, we set $\Omega_{1}=\left\{|u| \leq k ;\left|\frac{\partial}{\partial x_{i}} u_{\epsilon}\right| \leq 1\right\}$ and $\Omega_{2}=\left\{|u| \leq k ;\left|\frac{\partial}{\partial x_{i}} u_{\epsilon}\right|>1\right\}$, two subset of $\tilde{\Omega}$.

Using Proposition 3.1-(iii), we have

$$
\begin{aligned}
\sum_{i=1}^{N} \int_{\tilde{\Omega}}\left(\left|\frac{\partial}{\partial x_{i}} T_{k}\left(u_{\epsilon}\right)\right|^{p_{i}^{-}}\right) d x= & \sum_{i=1}^{N} \int_{\Omega_{1}}\left(\left|\frac{\partial}{\partial x_{i}} T_{k}\left(u_{\epsilon}\right)\right|^{p_{i}^{-}}\right) d x \\
& +\sum_{i=1}^{N} \int_{\Omega_{2}}\left(\left|\frac{\partial}{\partial x_{i}} T_{k}\left(u_{\epsilon}\right)\right|^{p_{i}^{-}}\right) d x \\
\leq & \operatorname{Nmeas}(\tilde{\Omega})+\sum_{i=1}^{N} \int_{\tilde{\Omega}}\left(\left|\frac{\partial}{\partial x_{i}} T_{k}\left(u_{\epsilon}\right)\right|^{p_{i}(x)}\right) d x \\
\leq & N \operatorname{meas}(\tilde{\Omega})+k\left(\|f\|_{L^{1}(\Omega)}+\|\tilde{d}\|_{L^{1}\left(\tilde{\Gamma}_{N e}\right)}\right) \leq C(k+1),
\end{aligned}
$$

with $C=\max \left\{N \operatorname{Neas}(\tilde{\Omega}) ;\left(\|f\|_{L^{1}(\Omega)}+\|\tilde{d}\|_{L^{1}\left(\tilde{\Gamma}_{N e}\right)}\right)\right\}$.

Lemma 3.6. Assume (1.4)-(1.8), $f \in L^{1}(\Omega)$ and $\tilde{d} \in L^{1}\left(\tilde{\Gamma}_{N e}\right)$. Let $u_{\epsilon}$ be a solution of the problem $P_{\epsilon}\left(\tilde{\rho}, \tilde{f}_{\epsilon}, \tilde{d}_{\epsilon}\right)$. For all $k>0$, there is two constants $C_{1}$ and $C_{2}$ such that 
(i) $\left\|u_{\epsilon}\right\|_{\mathcal{M}^{q^{*}(\tilde{\Omega})}} \leq C_{1}$;

(ii) $\left\|\frac{\partial}{\partial x_{i}} u_{\epsilon}\right\|_{\mathcal{M}^{p_{i}^{-} q / p}(\tilde{\Omega})} \leq C_{2}$.

Proof. (i) By Lemma 3.5, we have

$$
\sum_{i=1}^{N} \int_{\tilde{\Omega}}\left|\frac{\partial}{\partial x_{i}} T_{k}\left(u_{\epsilon}\right)\right|^{p_{i}^{-}} d x \leq C(1+k), \forall k>0 \text { and } i=1, \ldots, N .
$$

- If $k>1$, we have

$$
\sum_{i=1}^{N} \int_{\tilde{\Omega}}\left|\frac{\partial}{\partial x_{i}} T_{k}\left(u_{\epsilon}\right)\right|^{p_{i}^{-}} d x \leq C^{\prime} k,
$$

which means $T_{k}\left(u_{\epsilon}\right) \in W^{1,\left(p_{1}^{-}, \ldots, p_{N}^{-}\right)}(\tilde{\Omega})$.

Using relation (2.8), we deduce that

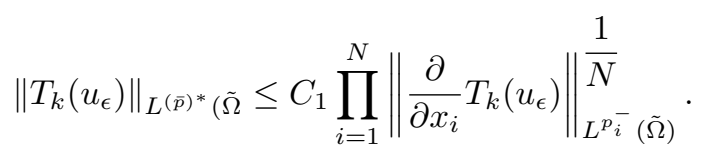

So,

$$
\begin{aligned}
\int_{\tilde{\Omega}}\left|T_{k}\left(u_{\epsilon}\right)\right|^{(\bar{p})^{*}} d x & \leq C\left[\prod_{i=1}^{N}\left(\int_{\tilde{\Omega}}\left|\frac{\partial}{\partial x_{i}} T_{k}\left(u_{\epsilon}\right)\right|^{p_{i}^{-}} d x\right)^{\frac{1}{N p_{i}^{-}}}\right]^{(\bar{p})^{*}} \\
& \leq C^{\prime \prime}\left[\prod_{i=1}^{N}(k)^{\frac{1}{N p_{i}^{-}}}\right]^{(\bar{p})^{*}} \\
& \leq C^{\prime \prime}\left[\sum_{k^{i=1}}^{N} \frac{1}{N p_{i}^{-}}\right]^{(\bar{p})^{*}} \\
& \leq C^{\prime \prime} k \frac{(\bar{p})^{*}}{\bar{p}} .
\end{aligned}
$$

Thus,

$$
\begin{aligned}
\int_{\left\{\left|u_{\epsilon}\right|>k\right\}}\left|T_{k}\left(u_{\epsilon}\right)\right|^{(\bar{p})^{*}} d x & \leq \int_{\tilde{\Omega}}\left|T_{k}\left(u_{\epsilon}\right)\right|^{(\bar{p})^{*}} d x \\
& \leq C^{\prime} k \frac{(\bar{p})^{*}}{\bar{p}}
\end{aligned}
$$

and so,

$$
(k)^{(\bar{p})^{*}} \operatorname{meas}\left\{x \in \tilde{\Omega}:\left|u_{\epsilon}\right|>k\right\} \leq C^{\prime} k^{\frac{(\bar{p})^{*}}{\bar{p}}}
$$

which means that

$$
\lambda_{u_{\epsilon}}(k) \leq C^{\prime} k^{(\bar{p})^{*}\left(\frac{1}{\bar{p}}-1\right)}=C^{\prime} k^{-q^{*}}, \forall k \geq 1 .
$$


- If $0<k<1$, we have

$$
\begin{aligned}
\lambda_{u_{\epsilon}}(k) & =\operatorname{meas}\left\{x \in \tilde{\Omega}:\left|u_{\epsilon}\right|>k\right\} \\
& \leq \operatorname{meas}(\tilde{\Omega}) \\
& \leq \operatorname{meas}(\tilde{\Omega}) k^{-q^{*}}
\end{aligned}
$$

So,

$$
\lambda_{u_{\epsilon}}(k) \leq\left(C^{\prime}+\operatorname{meas}(\tilde{\Omega})\right) k^{-q^{*}}=C_{1} k^{-q^{*}} .
$$

Therefore,

$$
\left\|u_{\epsilon}\right\|_{\mathcal{M}^{q^{*}}(\tilde{\Omega})} \leq C_{1} .
$$

(ii)• Let $\alpha \geq 1$. For all $k \geq 1$, we have

$$
\begin{aligned}
\lambda_{\frac{\partial u_{\epsilon}}{\partial x_{i}}}(\alpha) & =\operatorname{meas}\left(\left\{\left|\frac{\partial u_{\epsilon}}{\partial x_{i}}\right|>\alpha\right\}\right) \\
& =\operatorname{meas}\left(\left\{\left|\frac{\partial u_{\epsilon}}{\partial x_{i}}\right|>\alpha ;\left|u_{\epsilon}\right| \leq k\right\}\right)+\operatorname{meas}\left(\left\{\left|\frac{\partial u_{\epsilon}}{\partial x_{i}}\right|>\alpha ;\left|u_{\epsilon}\right|>k\right\}\right) \\
& \leq \int\left\{\left|\frac{\partial u_{\epsilon}}{\partial x_{i}}\right|>\alpha ;\left|u_{\epsilon}\right| \leq k\right\} \\
& \leq \int_{\left\{\left|u_{\epsilon}\right| \leq k\right\}}\left(\frac{1}{\alpha}\left|\frac{\partial u_{\epsilon}}{\partial x_{i}}\right|\right)^{p_{i}^{-}} d x+\lambda_{u_{\epsilon}}(k) \\
& \leq \alpha^{-p_{i}^{-}} C^{\prime} k+C k^{-q^{*}}(k) \\
& \leq B\left(\alpha^{-p_{i}^{-}} k+k^{-q^{*}}\right),
\end{aligned}
$$

with $B=\max \left(C^{\prime} ; C\right)$.

Let $g:[1 ; \infty) \rightarrow \mathbb{R}, x \mapsto g(x)=\frac{x}{\alpha^{p_{i}^{-}}}+x^{-q^{*}}$.

We have $g^{\prime}(x)=0$ with $x=\left(q^{*} \alpha^{p^{-}}\right) \frac{1}{q^{*}+1}$.

We set $k=\left(q^{*} \alpha^{p_{i}^{-}}\right)^{\frac{1}{q^{*}+1}} \geq 1$ in the above inequality to get,

$$
\begin{aligned}
\lambda_{\frac{\partial u_{\epsilon}}{\partial x_{i}}}(\alpha) & \leq B\left[\alpha^{-p_{i}^{-}} \times\left(q^{*} \alpha^{p_{i}^{-}}\right) \frac{1}{q^{*}+1}+\left(q^{*} \alpha^{p_{i}^{-}}\right) \frac{-q^{*}}{q^{*}+1}\right] \\
& \leq B\left[\left(q^{*}\right)^{\frac{1}{q^{*}+1}} \times \alpha^{-p_{i}^{-}\left(1-\frac{1}{q^{*}+1}\right)}+\left(q^{*}\right)^{\frac{-q^{*}}{q^{*}+1}} \times \alpha^{\frac{-p_{i}^{-} q^{*}}{q^{*}+1}}\right]
\end{aligned}
$$




$$
\begin{aligned}
& \leq B\left[\left(q^{*}\right)^{\frac{1}{q^{*}+1}} \times \alpha^{-p_{i}^{-}\left(\frac{q^{*}}{q^{*}+1}\right)}+\left(q^{*}\right)^{\frac{-q^{*}}{q^{*}+1}} \times \alpha \frac{-p_{i}^{-} q^{*}}{q^{*}+1}\right] \\
& \leq M \alpha^{-p_{i}^{-}} \frac{q^{*}}{q^{*}+1} \\
& \leq M \alpha^{-p_{i}^{-}} \frac{q}{\bar{p}}
\end{aligned}
$$

where $M=B \times \max \left(\left(q^{*}\right)^{\frac{1}{q^{*}+1}} ;\left(q^{*}\right)^{\frac{-q^{*}}{q^{*}+1}}\right)$ and as $q^{*}=\frac{N(\bar{p}-1)}{N-\bar{p}}, q=\frac{N(\bar{p}-1)}{N-1}$.

So,

$$
\begin{aligned}
\frac{q^{*}}{q^{*}+1} & =\frac{q^{*}(N-\bar{p})}{N(\bar{p}-1)+N-\bar{p}} \\
& =\frac{q^{*}(N-\bar{p})}{N \bar{p}-\bar{p}} \\
& =\frac{N(\bar{p}-1)}{(N-1) \bar{p}} \\
& =\frac{q}{\bar{p}} .
\end{aligned}
$$

- If $0 \leq \alpha<1$, we have.

$$
\begin{aligned}
\lambda_{\frac{\partial u_{\epsilon}}{\partial x_{i}}}(\alpha) & =\operatorname{meas}\left(\left\{x \in \tilde{\Omega}:\left|\frac{\partial u_{\epsilon}}{\partial x_{i}}\right|>\alpha\right\}\right) \\
& \leq \operatorname{meas}(\tilde{\Omega}) \alpha^{-p_{i}^{-}} \frac{q}{\bar{p}}
\end{aligned}
$$

Therefore,

$$
\lambda_{\frac{\partial u_{\epsilon}}{\partial x_{i}}}(\alpha) \leq(M+\operatorname{meas}(\tilde{\Omega})) \alpha^{-p_{i}^{-}} \frac{q}{\bar{p}}, \forall \alpha \geq 0
$$

So,

$$
\left\|\frac{\partial u_{\epsilon}}{\partial x_{i}}\right\|_{H} \leq C_{2}
$$

where $H=\mathcal{M}(\tilde{\Omega}) \frac{p_{i}^{-} q}{\bar{p}}$

Proposition 3.2. Assume (1.4)-(1.8), $f \in L^{1}(\Omega)$ and $\tilde{d} \in L^{1}\left(\tilde{\Gamma}_{N e}\right)$. Let $u_{\epsilon}$ be a solution of the problem $P_{\epsilon}\left(\tilde{\rho}, \tilde{f}_{\epsilon}, \tilde{d}_{\epsilon}\right)$. Then,

(i) $u_{\epsilon} \rightarrow u$ in measure, a.e. in $\Omega$ and a.e. on $\tilde{\Gamma}_{N e}$;

(ii) For all $i=1, \ldots N, \frac{\partial T_{k}\left(u_{\epsilon}\right)}{\partial x_{i}} \rightarrow \frac{\partial T_{k}(u)}{\partial x_{i}}=0$ in $L^{p_{i}^{-}}(\tilde{\Omega} \backslash \Omega)$.

Proof. (i) By Proposition $3.1(i)$, we deduce that $\left(T_{k}\left(u_{\epsilon}\right)\right)_{\epsilon>0}$ is bounded in $W_{D}^{1, \vec{p}(.)}(\tilde{\Omega}) \hookrightarrow L^{p_{m}(.)}(\tilde{\Omega}) \hookrightarrow$ $L^{p_{m}^{-}}(\tilde{\Omega})$ compact. Therefore, up to a subsequence, we can assume that as $\epsilon \rightarrow 0,\left(T_{k}\left(u_{\epsilon}\right)\right)_{\epsilon>0}$ converges strongly to some function in $L^{p_{m}^{-}}(\tilde{\Omega})$, a.e. in $\tilde{\Omega}$ and a.e. on $\tilde{\Gamma}_{N e}$.

Let us see that the sequence $\left(u_{\epsilon}\right)_{\epsilon>0}$ is Cauchy in measure. 
Indeed, let $s>0$ and define:

$E_{1}=\left[\left|u_{\epsilon_{1}}\right|>k\right], E_{2}=\left[\left|u_{\epsilon_{2}}\right|>k\right]$ and $E_{3}=\left[\left|T_{k}\left(u_{\epsilon_{1}}\right)-T_{k}\left(u_{\epsilon_{2}}\right)\right|>s\right]$,

where $k>0$ is fixed. We note that

$$
\left[\left|u_{\epsilon_{1}}-u_{\epsilon_{2}}\right|>s\right] \subset E_{1} \cup E_{2} \cup E_{3} ;
$$

hence,

$$
\operatorname{meas}\left(\left[\left|u_{\epsilon_{1}}-u_{\epsilon_{2}}\right|>s\right]\right) \leq \sum_{i=1}^{3} E_{i} .
$$

Let $\theta>0$, using Lemma 3.4, we choose $k=k(\theta)$ such that

$$
\operatorname{meas}\left(E_{1}\right) \leq \frac{\theta}{3} \text { and } \operatorname{meas}\left(E_{2}\right) \leq \frac{\theta}{3} .
$$

Since $\left(T_{k}\left(u_{\epsilon}\right)\right)_{\epsilon>0}$ converges strongly in $L^{p_{m}^{-}}(\tilde{\Omega})$, then, it is a Cauchy sequence in $L^{p_{m}^{-}}(\tilde{\Omega})$.

Thus,

$$
\operatorname{meas}\left(E_{3}\right) \leq \frac{1}{s^{p_{m}^{-}}} \int_{\Omega}\left|T_{k}\left(u_{\epsilon_{1}}\right)-T_{k}\left(u_{\epsilon_{2}}\right)\right|^{p_{m}^{-}} d x \leq \frac{\theta}{3},
$$

for all $\epsilon_{1}, \epsilon_{2} \geq n_{0}(s, \theta)$. Finally, from (3.26), (3.27) and (3.28), we obtain

$$
\operatorname{meas}\left(\left[\left|u_{\epsilon_{1}}-u_{\epsilon_{2}}\right|>s\right]\right) \leq \theta \text { for all } \epsilon_{1}, \epsilon_{2} \geq n_{0}(s, \theta)
$$

which means that the sequence $\left(u_{\epsilon}\right)_{\epsilon>0}$ is Cauchy in measure, so $u_{\epsilon} \rightarrow u$ in measure and up to a subsequence, we have $u_{\epsilon} \rightarrow u$ a.e. in $\tilde{\Omega}$. Hence, $\sigma_{k}=T_{k}(u)$ a.e. in $\tilde{\Omega}$ and so, $u \in \mathcal{T}_{D}^{1, \vec{p}(.)}(\Omega)$.

(ii) According to the proof of (i), we have $T_{k}\left(u_{\epsilon}\right) \rightarrow T_{k}(u)$ in $W_{D}^{1, \vec{p}(.)}(\tilde{\Omega}) \hookrightarrow W_{D}^{1, \vec{p}_{-}}(\tilde{\Omega})$ which implies on one hand that for all $i=1, \ldots N, \frac{\partial T_{k}\left(u_{\epsilon}\right)}{\partial x_{i}} \rightarrow \frac{\partial T_{k}(u)}{\partial x_{i}}$ in $L^{p_{i}^{-}}(\tilde{\Omega})$ and on the other hand that for all $i=1, \ldots N$, $\frac{\partial T_{k}\left(u_{\epsilon}\right)}{\partial x_{i}} \rightarrow \frac{\partial T_{k}(u)}{\partial x_{i}}$ in $L^{p_{i}^{-}}(\tilde{\Omega})$

and then for all $i=1, \ldots N, \frac{\partial T_{k}\left(u_{\epsilon}\right)}{\partial x_{i}} \rightarrow \frac{\partial T_{k}(u)}{\partial x_{i}}$ in $L^{p_{i}^{-}}(\tilde{\Omega} \backslash \Omega)$.

Let $i=1, \ldots, N$, by Proposition 3.1- $(i)$, we can assert $\left(\frac{1}{\epsilon} \frac{\partial T_{k}\left(u_{\epsilon}\right)}{\partial x_{i}}\right)$ is bounded in $L^{p_{i}^{-}}(\tilde{\Omega} \backslash \Omega)$. Indeed, let $k>0$, we set $\Omega^{1}=\left\{x \in \tilde{\Omega} \backslash \Omega ;|u(x)| \leq k ;\left|\frac{\partial}{\partial x_{i}} u_{\epsilon}(x)\right| \leq \epsilon\right\}$ and $\Omega^{2}=\left\{x \in \tilde{\Omega} \backslash \Omega ;|u| \leq k ;\left|\frac{\partial}{\partial x_{i}} u_{\epsilon}(x)\right|>\epsilon\right\} ;$ using Proposition 3.1-(i), we have

$$
\begin{aligned}
\sum_{i=1}^{N} \int_{\tilde{\Omega} \backslash \Omega}\left(\frac{1}{\epsilon}\left|\frac{\partial T_{k}\left(u_{\epsilon}\right)}{\partial x_{i}}\right|^{p_{i}^{-}}\right) d x & =\sum_{i=1}^{N} \int_{\Omega^{1}}\left(\frac{1}{\epsilon}\left|\frac{\partial T_{k}\left(u_{\epsilon}\right)}{\partial x_{i}}\right|^{p_{i}^{-}}\right) d x+ \\
& \sum_{i=1}^{N} \int_{\Omega^{2}}\left(\frac{1}{\epsilon}\left|\frac{\partial}{\partial x_{i}} T_{k}\left(u_{\epsilon}\right)\right|^{p_{i}^{-}}\right) d x \\
\leq & N \operatorname{meas}(\tilde{\Omega} \backslash \Omega)+\sum_{i=1}^{N} \int_{\tilde{\Omega} \backslash \Omega}\left(\frac{1}{\epsilon}\left|\frac{\partial}{\partial x_{i}} T_{k}\left(u_{\epsilon}\right)\right|^{p_{i}(x)}\right) d x \\
\leq & N \operatorname{meas}(\tilde{\Omega} \backslash \Omega)+k\left(\|f\|_{L^{1}(\Omega)}+\|\tilde{d}\|_{L^{1}\left(\tilde{\Gamma}_{N e}\right)}\right) \\
\leq & C^{\prime}(k+1),
\end{aligned}
$$


with $C^{\prime}=\max \left\{N \operatorname{meas}(\tilde{\Omega} \backslash \Omega) ;\left(\|f\|_{L^{1}(\Omega)}+\|\tilde{d}\|_{L^{1}\left(\tilde{\Gamma}_{N e}\right)}\right)\right\}$.

To end,

$$
\int_{\tilde{\Omega} \backslash \Omega}\left(\frac{1}{\epsilon}\left|\frac{\partial T_{k}\left(u_{\epsilon}\right)}{\partial x_{i}}\right|^{p_{i}^{-}}\right) d x \leq \sum_{i=1}^{N} \int_{\tilde{\Omega} \backslash \Omega}\left(\frac{1}{\epsilon}\left|\frac{\partial T_{k}\left(u_{\epsilon}\right)}{\partial x_{i}}\right|^{p_{i}^{-}}\right) d x, \text { for any } \mathrm{i}=1, \ldots, \mathrm{N} .
$$

Therefore, there exists $\Theta_{k} \in L^{p_{i}^{-}}(\tilde{\Omega} \backslash \Omega)$ such that

$$
\frac{1}{\epsilon} \frac{\partial T_{k}\left(u_{\epsilon}\right)}{\partial x_{i}} \rightarrow \Theta_{k} \text { in } L^{p_{i}^{-}}(\tilde{\Omega} \backslash \Omega) \text { as } \epsilon \rightarrow 0 .
$$

For any $\psi \in L^{\left(p_{i}^{\prime}\right)^{-}}(\tilde{\Omega} \backslash \Omega)$, we have

$$
\int_{\tilde{\Omega} \backslash \Omega} \frac{\partial T_{k}\left(u_{\epsilon}\right)}{\partial x_{i}} \psi d x=\int_{\tilde{\Omega} \backslash \Omega}\left(\frac{1}{\epsilon} \frac{\partial T_{k}\left(u_{\epsilon}\right)}{\partial x_{i}}-\Theta_{k}\right)(\epsilon \psi) d x+\epsilon \int_{\tilde{\Omega} \backslash \Omega} \Theta_{k} \psi d x .
$$

As $(\epsilon \psi)_{\epsilon>0}$ converges strongly to zero in $L^{\left(p^{\prime}\right)^{-}}(\tilde{\Omega} \backslash \Omega)$, we pass to the limit as $\epsilon \rightarrow 0$ in (3.30), to get

$$
\frac{\partial T_{k}\left(u_{\epsilon}\right)}{\partial x_{i}} \rightarrow 0 \text { in } L^{p_{i}^{-}}(\tilde{\Omega} \backslash \Omega) .
$$

Hence, one has

$$
\frac{\partial T_{k}\left(u_{\epsilon}\right)}{\partial x_{i}} \rightarrow \frac{\partial T_{k}(u)}{\partial x_{i}}=0 \text { in } L^{p_{i}^{-}}(\tilde{\Omega} \backslash \Omega)
$$

for any $i=1, \ldots, N \square$

Lemma 3.7. $b(u) \in L^{1}(\Omega)$ and $\tilde{\rho}(u) \in L^{1}\left(\tilde{\Gamma}_{N e}\right)$.

Proof. Having in mind that by Proposition 3.1-(ii),

$$
\int_{\Omega}\left|b\left(u_{\epsilon}\right)\right| d x+\int_{\tilde{\Gamma}_{N e}}\left|\tilde{\rho}\left(u_{\epsilon}\right)\right| d \sigma \leq\left(\|f\|_{L^{1}(\Omega)}+\|\tilde{d}\|_{L^{1}\left(\tilde{\Gamma}_{N e}\right)}\right) .
$$

We deduce that

$$
\int_{\Omega}\left|b\left(u_{\epsilon}\right)\right| d x \leq\left(\|f\|_{L^{1}(\Omega)}+\|\tilde{d}\|_{L^{1}\left(\tilde{\Gamma}_{N e}\right)}\right)
$$

and

$$
\int_{\tilde{\Gamma}_{N e}}\left|\tilde{\rho}\left(u_{\epsilon}\right)\right| d \sigma \leq\left(\|f\|_{L^{1}(\Omega)}+\|\tilde{d}\|_{L^{1}\left(\tilde{\Gamma}_{N e}\right)}\right) .
$$

By Fatou's Lemma, the continuity of $b, \tilde{\rho}$ and using Proposition 3.2, we have

$$
\begin{gathered}
\liminf _{\epsilon \rightarrow 0} \int_{\Omega}\left|b\left(u_{\epsilon}\right)\right| d x \geq \int_{\Omega}|b(u)| d x, \\
\liminf _{\epsilon \rightarrow 0} \int_{\tilde{\Gamma}_{N e}}\left|\tilde{\rho}\left(u_{\epsilon}\right)\right| d \sigma \geq \int_{\tilde{\Gamma}_{N e}}|\tilde{\rho}(u)| d \sigma .
\end{gathered}
$$

Using (3.31)-(3.34), we deduce that

$$
\int_{\Omega}|b(u)| d x \leq\left(\|f\|_{L^{1}(\Omega)}+\|\tilde{d}\|_{L^{1}\left(\tilde{\Gamma}_{N e}\right)}\right)
$$

and

$$
\int_{\tilde{\Gamma}_{N e}}|\tilde{\rho}(u)| d \sigma \leq\left(\|f\|_{L^{1}(\Omega)}+\|\tilde{d}\|_{L^{1}\left(\tilde{\Gamma}_{N e}\right)}\right) .
$$

Therefore, $b(u) \in L^{1}(\Omega)$ and $\tilde{\rho}(u) \in L^{1}\left(\tilde{\Gamma}_{N e}\right)$. 
Lemma 3.8. Assume (1.4)-(1.8) hold and $u_{\epsilon}$ be a weak solution of the problem $P_{\epsilon}\left(\tilde{\rho}, \tilde{f}_{\epsilon}, \tilde{d}_{\epsilon}\right)$. Then,

(i) $\frac{\partial}{\partial x_{i}} u_{\epsilon}$ converges in measure to $\frac{\partial}{\partial x_{i}} u$.

(ii) $a_{i}\left(x, \frac{\partial T_{k}\left(u_{\epsilon}\right)}{\partial x_{i}}\right) \rightarrow a_{i}\left(x, \frac{\partial T_{k}(u)}{\partial x_{i}}\right)$ strongly in $L^{1}(\Omega)$ and weakly in $L^{p_{i}^{\prime}(.)}(\Omega)$, for all $i=1, \ldots, N$.

In order to give the proof of Lemma 3.8, we need the following lemmas.

Lemma 3.9 (Cf [8]). Let $u \in \mathcal{T}^{1, \vec{p}(.)}(\Omega)$. Then, there exists a unique measurable function $\nu_{i}: \Omega \rightarrow \mathbb{R}$ such that

$$
\nu_{i} \chi_{\{|u|<k\}}=\frac{\partial}{\partial x_{i}} T_{k}(u) \text { for a.e. } x \in \Omega, \forall k>0 \text { and } i=1, \ldots, N ;
$$

where $\chi_{A}$ denotes the characteristic function of a measurable set $A$.

The functions $\nu_{i}$ are denoted $\frac{\partial}{\partial x_{i}} u$. Moreover, if u belongs to $W^{1, \vec{p}(.)}(\Omega)$, then $v_{i} \in L^{p_{i}(.)}(\Omega)$ and coincides with the standard distributional gradient of u i.e. $\nu_{i}=\frac{\partial}{\partial x_{i}} u$.

Lemma 3.10 (Cf [11], lemma 5.4). Let $\left(v_{n}\right)_{n \in \mathbb{N}}$ be a sequence of measurable functions. If $v_{n}$ converges in measure to $v$ and is uniformly bounded in $L^{p(.)}(\Omega)$ for some $1<<p(.) \in L^{\infty}(\Omega)$, then $v_{n} \rightarrow v$ strongly in $L^{1}(\Omega)$.

The third technical lemma is a standard fact in measure theory ( $\mathrm{Cf}[7])$.

Lemma 3.11. Let $(X, \mathcal{M}, \mu)$ be a measurable space such that $\mu(X)<\infty$.

Consider a measurable function $\gamma: X \rightarrow[0 ; \infty]$ such that

$$
\mu(\{x \in X: \gamma(x)=0\})=0 .
$$

Then, for every $\epsilon>0$, there exists $\delta$ such that

$$
\mu(A))<\epsilon, \text { for all } A \in \mathcal{M} \text { with } \int_{A} \gamma d x<\delta .
$$

Proof of Lemma 3.8. (i) We claim that $\left(\frac{\partial}{\partial x_{i}} u_{\epsilon}\right)_{\epsilon \in \mathbb{N}}$ is Cauchy in measure. Indeed, let $s>0$, consider

$$
\begin{aligned}
& A_{n, m}:=\left\{\left|\frac{\partial}{\partial x_{i}} u_{n}\right|>h\right\} \cup\left\{\left|\frac{\partial}{\partial x_{i}} u_{m}\right|>h\right\}, \\
& B_{n, m}:=\left\{\left|u_{n}-u_{m}\right|>k\right\} \text { and } \\
& C_{n, m}:=\left\{\left|\frac{\partial}{\partial x_{i}} u_{n}\right| \leq h,\left|\frac{\partial}{\partial x_{i}} u_{m}\right| \leq h,\left|u_{n}-u_{m}\right| \leq k,\left|\frac{\partial}{\partial x_{i}} u_{n}-\frac{\partial}{\partial x_{i}} u_{m}\right|>s\right\}, \text { where } h \text { and } k \text { will be }
\end{aligned}
$$
chosen later. One has

$$
\left\{\left|\frac{\partial}{\partial x_{i}} u_{n}-\frac{\partial}{\partial x_{i}} u_{m}\right|>s\right\} \subset A_{n, m} \cup B_{n, m} \cup C_{n, m} .
$$

Let $\vartheta>0$. By Lemma 3.6, we can choose $h=h(\vartheta)$ large enough such that meas $\left(A_{n, m}\right) \leq \frac{\vartheta}{3}$ for all $n, m \geq 0$. On the other hand, by Proposition 3.2, we have that meas $\left(B_{n, m}\right) \leq \frac{\vartheta}{3}$ for all $n, m \geq n_{0}(k, \vartheta)$. Moreover, by assumption $\left(H_{3}\right)$, there exists a real valued function $\gamma: \Omega \rightarrow[0, \infty]$ such that meas $\{x \in \Omega: \gamma(x)=0\}=0$ and

$$
\left(a_{i}(x, \xi)-a_{i}\left(x, \xi^{\prime}\right)\right) \cdot\left(\xi-\xi^{\prime}\right) \geq \gamma(x),
$$

for all $i=1, \ldots, N,|\xi|,\left|\xi^{\prime}\right| \leq h,\left|\xi-\xi^{\prime}\right| \geq s$, for a.e. $x \in \Omega$. Indeed, let's set $K=\left\{(\xi, \eta) \in \mathbb{R}^{N} \times \mathbb{R}^{N}:|\xi| \leq\right.$ $h,|\eta| \leq h,|\xi-\eta| \geq s\}$. We have $K \subset B(0, h) \times B(0, h)$ and so $K$ is a compact set because it is closed in a 
compact set.

For all $x \in \Omega$ and for all $i=1, \ldots, N$, let us define $\phi: K \rightarrow[0 ; \infty]$ such that

$$
\psi(\xi, \eta)=\left(a_{i}(x, \xi)-a_{i}(x, \eta)\right) \cdot(\xi-\eta) .
$$

As for a.e. $x \in \Omega, a_{i}(x,$.$) is continuous on \mathbb{R}^{N}, \psi$ is continuous on the compact $K$, by Weierstrass theorem, there exists $\left(\xi_{0}, \eta_{0}\right) \in K$ such that

$$
\forall(\xi, \eta) \in K, \psi(\xi, \eta) \geq \psi\left(\xi_{0}, \eta_{0}\right)
$$

Now let us define $\gamma$ on $\Omega$ as follows.

$$
\gamma(x)=\psi_{i}\left(\xi_{0}, \eta_{0}\right)=\left(a_{i}\left(x, \xi_{0}\right)-a_{i}(x, \eta)\right) \cdot\left(\xi-\eta_{0}\right) .
$$

Since $s>0$, the function $\gamma$ is such that meas $(\{x \in \Omega: \gamma(x)=0\})=0$. Let $\delta=\delta(\epsilon)$ be given by Lemma 3.11, replacing $\epsilon$ and $A$ by $\frac{\epsilon}{3}$ and $C_{n, m}$ respectively. Taking respectively $\tilde{\xi}=T_{k}\left(u_{n}-u_{m}\right)$ and $\tilde{\xi}=T_{k}\left(u_{m}-u_{n}\right)$ for the weak solution $u_{n}$ and $u_{m}$ in (3.19) and after adding the two relations, we have

$$
\left\{\begin{array}{l}
\sum_{i=1}^{N} \int_{\left\{\left|u_{n}-u_{m}\right|<k\right\}}\left(a_{i}\left(x, \frac{\partial}{\partial x_{i}} u_{n}\right)-a_{i}\left(x, \frac{\partial}{\partial x_{i}} u_{m}\right)\right)\left(\frac{\partial}{\partial x_{i}}\left(u_{n}-u_{m}\right)\right) d x \\
+\int_{Q}\left(\left(\frac{1}{\epsilon^{p_{i}(x)}}\left|\frac{\partial u_{n}}{\partial x_{i}}\right|^{p_{i}(x)-2} \frac{\partial u_{n}}{\partial x_{i}}\right)-\left(\frac{1}{\epsilon^{p_{i}(x)}}\left|\frac{\partial u_{m}}{\partial x_{i}}\right|^{p_{i}(x)-2} \frac{\partial u_{m}}{\partial x_{i}}\right)\left(\frac{\partial\left(u_{n}-u_{m}\right)}{\partial x_{i}}\right)\right) d x \\
+\int_{\Omega}\left(\left|u_{n}\right|^{p_{M}(x)-2} u_{n}-\left.u_{m}\right|^{p_{M}(x)-2} u_{m}\right)\left(T_{k}\left(u_{n}-u_{m}\right) d x\right. \\
+\int_{\tilde{\Gamma}_{N e}}\left(\tilde{\rho}\left(u_{n}\right)-\tilde{\rho}\left(u_{m}\right)\right) T_{k}\left(u_{n}-u_{m}\right) d \sigma \\
=2\left(\int_{\Omega} f_{\epsilon} T_{k}\left(u_{n}-u_{m}\right) d x+\int_{\tilde{\Gamma}_{N e}} \tilde{d}_{\epsilon} T_{k}\left(u_{n}-u_{m}\right) d \sigma\right),
\end{array}\right.
$$

where $Q=\left\{\tilde{\Omega} \backslash \Omega \cap\left\{\left|u_{n}-u_{m}\right|<k\right\}\right\}$. As the three last terms on the left hand side are non-negative and

$$
\int_{\Omega} f_{\epsilon} T_{k}\left(u_{n}-u_{m}\right) d x+\int_{\tilde{\Gamma}_{N e}} \tilde{d}_{\epsilon} T_{k}\left(u_{n}-u_{m}\right) d \sigma \leq k\left(\|f\|_{L^{1}(\Omega)}+\|\tilde{d}\|_{L^{1}\left(\tilde{\Gamma}_{N e}\right)}\right),
$$

we deduce that

$$
\left\{\begin{array}{l}
\sum_{i=1}^{N} \int_{\left\{\left|u_{n}-u_{m}\right|<k\right\}}\left(a_{i}\left(x, \frac{\partial u_{n}}{\partial x_{i}}\right)-a_{i}\left(x, \frac{\partial u_{m}}{\partial x_{i}}\right)\right)\left(\frac{\partial\left(u_{n}-u_{m}\right)}{\partial x_{i}}\right) d x \\
\leq 2 k\left(\|f\|_{L^{1}(\Omega)}+\|\tilde{d}\|_{L^{1}\left(\tilde{\Gamma}_{N e}\right)}\right) .
\end{array}\right.
$$

Therefore, using $\left(H_{3}\right)$ we have

$$
\begin{aligned}
\int_{C_{n, m}} \gamma d x & \leq \int_{C_{n, m}}\left(a_{i}\left(x, \frac{\partial}{\partial x_{i}} u_{n}\right)-a_{i}\left(x, \frac{\partial}{\partial x_{i}} u_{m}\right)\right) \frac{\partial}{\partial x_{i}}\left(u_{n}-u_{m}\right) d x \\
& \leq \sum_{i=1}^{N} \int_{C_{n, m}}\left(a_{i}\left(x, \frac{\partial}{\partial x_{i}} u_{n}\right)-a_{i}\left(x, \frac{\partial}{\partial x_{i}} u_{m}\right)\right) \frac{\partial}{\partial x_{i}}\left(u_{n}-u_{m}\right) d x \\
& \leq 2 k\left(\|\tilde{d}\|_{L^{1}\left(\tilde{\Gamma}_{N e}\right)}+\|f\|_{L^{1}(\Omega)}\right)<\delta
\end{aligned}
$$

by choosing $k=\delta / 4\left(\|\tilde{d}\|_{L^{1}\left(\tilde{\Gamma}_{N e}\right)}+\|f\|_{L^{1}(\Omega)}\right)$. From Lemma 3.11 again, it follows that meas $\left(C_{n, m}\right)<\frac{\vartheta}{3}$. Thus, using (3.36) and the estimates obtained for $A_{n, m}, B_{n, m}$ and $C_{n, m}$, it follows that

$$
\text { meas }\left(\left\{\left|\frac{\partial}{\partial x_{i}} u_{n}-\frac{\partial}{\partial x_{i}} u_{m}\right|>s\right\}\right) \leq \vartheta
$$


for all $n, m \geq n_{0}(s, \vartheta)$, and then the claim is proved.

As consequence, $\left(\frac{\partial}{\partial x_{i}} u_{\epsilon}\right)_{\epsilon \in \mathbb{N}}$ converges in measure to some measurable function $\nu_{i}$.

In order to end the proof of Lemma 3.8, we need the following lemma.

Lemma 3.12. (a) For a.e. $k \in \mathbb{R}, \frac{\partial}{\partial x_{i}} T_{k}\left(u_{\epsilon}\right)$ converges in measure to $\nu_{i} \chi_{\{|u|<k\}}$.

(b) For a.e. $k \in \mathbb{R}, \frac{\partial}{\partial x_{i}} T_{k}(u)=\nu_{i} \chi_{\{|u|<k\}}$.

(c) $\frac{\partial}{\partial x_{i}} T_{k}(u)=\nu_{i} \chi_{\{|u|<k\}}$ holds for all $k \in \mathbb{R}$.

Proof. (a) We know that $\frac{\partial}{\partial x_{i}} u_{\epsilon} \rightarrow \nu_{i}$ in measure. Thus $\frac{\partial}{\partial x_{i}} u_{\epsilon} \chi_{\{|u|<k\}} \rightarrow \nu_{i} \chi_{\{|u|<k\}}$ in measure.

Now, let us show that $\left(\chi_{\left\{\left|u_{\epsilon}\right|<k\right\}}-\chi_{\{|u|<k\}}\right) \frac{\partial}{\partial x_{i}} u_{\epsilon} \rightarrow 0$ in measure.

For that, it is sufficient to show that $\left(\chi_{\left\{\left|u_{\epsilon}\right|<k\right\}}-\chi_{\{|u|<k\}}\right) \rightarrow 0$ in measure. Now, for all $\delta>0$, $\left\{\left|\chi_{\left\{\left|u_{\epsilon}\right|<k\right\}}-\chi_{\{|u|<k\}}\right|\left|\frac{\partial}{\partial x_{i}} u_{\epsilon}\right|>\delta\right\} \subset\left\{\left|\chi_{\left\{\left|u_{\epsilon}\right|<k\right\}}-\chi_{\{|u|<k\}}\right| \neq 0\right\} \subset\{|u|=k\} \cup\left\{u_{\epsilon}<k<\right.$ $u\} \cup\left\{u<k<u_{\epsilon}\right\} \cup\left\{u_{\epsilon}<-k<u\right\} \cup\left\{u<-k<u_{\epsilon}\right\}$. Thus,

$$
\left\{\begin{aligned}
\operatorname{meas}\left(\left\{\left|\chi_{\left\{\left|u_{\epsilon}\right|<k\right\}}-\chi_{\{|u|<k\}}\right|\left|\frac{\partial}{\partial x_{i}} u_{\epsilon}\right|>\delta\right\}\right) \leq & \text { meas }(\{|u|=k\}) \\
& + \text { meas }\left(\left\{u_{\epsilon}<k<u\right\}\right) \\
& + \text { meas }\left(\left\{u<k<u_{\epsilon}\right\}\right) \\
& + \text { meas }\left(\left\{u_{\epsilon}<-k<u\right\}\right) \\
& + \text { meas }\left(\left\{u<-k<u_{\epsilon}\right\}\right) .
\end{aligned}\right.
$$

Note that

$\left\{\begin{array}{l}\text { meas }(\{|u|=k\}) \leq \text { meas }(\{k-h<u<k+h\}) \\ + \text { meas }(\{-k-h<u<-k+h\}) \rightarrow 0 \text { as } h \rightarrow 0 \text { for a.e. } k>0\end{array}\right.$, since $u$ is fixed function.

Next, meas $\left(\left\{u_{\epsilon}<k<u\right\}\right) \leq$ meas $(\{k<u<k+h\})+$ meas $\left(\left\{\left|u_{\epsilon}-u\right|>h\right\}\right)$, for all $h>0$.

Due to Proposition 3.2, we have for all fixed $h>0$, meas $\left(\left\{\left|u_{\epsilon}-u\right|>h\right\}\right) \rightarrow 0$ as $\epsilon \rightarrow 0$. Since meas $(\{k<u<k+h\}) \rightarrow 0$ as $h \rightarrow 0$, for all $\vartheta>0$, one can find $N$ such that for all $n>N$, meas $(\{|u|=k\})<\frac{\vartheta}{2}+\frac{\vartheta}{2}=\vartheta$ by choosing $h$ and then $N$. Each of the other terms on the right-hand side of (3.38) can be treated in the same way as for meas $\left(\left\{u_{\epsilon}<k<u\right\}\right)$. Thus, meas $\left(\left\{\left|\chi_{\left\{\left|u_{\epsilon}\right|<k\right\}}-\chi_{\{|u|<k\}}\right|\left|\frac{\partial}{\partial x_{i}} u_{\epsilon}\right|>\delta\right\}\right) \rightarrow 0$ as $\epsilon \rightarrow 0$. Finally, since $\frac{\partial}{\partial x_{i}} T_{k}\left(u_{\epsilon}\right)=$ $\frac{\partial}{\partial x_{i}} u_{\epsilon} \chi_{\left\{\left|u_{\epsilon}\right|<k\right\}},(a)$ follows.

(b) Using the Proof of Proposition 3.2-(ii) we have $\frac{\partial}{\partial x_{i}} T_{k}\left(u_{\epsilon}\right) \rightarrow \frac{\partial}{\partial x_{i}} T_{k}(u)$ weakly in $L^{p_{i}^{-}}(\tilde{\Omega})$. The previous convergence also ensures that $\frac{\partial}{\partial x_{i}} T_{k}\left(u_{\epsilon}\right)$ converges to $\frac{\partial}{\partial x_{i}} T_{k}(u)$ weakly in $L^{1}(\Omega)$. On the other hand, by $(a), \frac{\partial}{\partial x_{i}} T_{k}\left(u_{\epsilon}\right)$ converges to $\nu_{i} \chi_{|u|<k\}}$ in measure. By Lemma 3.10, since $\frac{\partial}{\partial x_{i}} T_{k}\left(u_{\epsilon}\right)$ is uniformly bounded in $L^{p_{i}^{-}}(\tilde{\Omega})$ (see Lemma 3.5) hence in $L^{p_{i}^{-}}(\Omega)$, the convergence is actually strong in $L^{1}(\Omega)$; thus it is also weak in $L^{1}(\Omega)$. By the uniqueness of a weak $L^{1}$-limit, $\nu_{i} \chi_{\{|u|<k\}}$ coincides with $\frac{\partial}{\partial x_{i}} T_{k}(u)$. 
(c) Let $0<k<s$, and $s$ be such that $\nu_{i} \chi_{\{|u|<s\}}$ coincides with $\frac{\partial}{\partial x_{i}} T_{s}(u)$. Then,

$$
\begin{aligned}
\frac{\partial}{\partial x_{i}} T_{k}(u) & =\frac{\partial}{\partial x_{i}} T_{k}\left(T_{s}(u)\right) \\
& =\frac{\partial}{\partial x_{i}} T_{s}(u) \chi_{\left\{\left|T_{s}(u)\right|<k\right\}} \\
& =\nu_{i} \chi_{\{|u|<s\}} \chi_{\{|u|<k\}} \\
& =\nu_{i} \chi_{\{|u|<k\}} .
\end{aligned}
$$

Now, we can end the proof of Lemma 3.8. Indeed, combining lemmas 3.12 (c) and 3.9; (i) follows.

Next, by lemmas 3.10 and 3.12, we have for all $k>0, i=1, \ldots, N, a_{i}\left(x, \frac{\partial}{\partial x_{i}} T_{k}\left(u_{\epsilon}\right)\right)$ converges to $a_{i}\left(x, \frac{\partial}{\partial x_{i}} T_{k}(u)\right)$ in $L^{1}(\Omega)$ strongly. Indeed, Let $s, k>0$, consider

$E_{4}=\left\{\left|\frac{\partial u_{n}}{\partial x_{i}}-\frac{\partial u_{m}}{\partial x_{i}}\right|>s,\left|u_{n}\right| \leq k,\left|u_{m}\right| \leq k\right\}$,

$E_{5}=\left\{\left|\frac{\partial u_{m}}{\partial x_{i}}\right|>s,\left|u_{n}\right|>k,\left|u_{m}\right| \leq k\right\}$ and

$E_{6}=\left\{\left|\frac{\partial u_{n}}{\partial x_{i}}\right|>s,\left|u_{n}\right| \leq k,\left|u_{m}\right|>k\right\}$.

We have

$$
\left\{\left|\frac{\partial T_{k}\left(u_{n}\right)}{\partial x_{i}}-\frac{\partial T_{k}\left(u_{m}\right)}{\partial x_{i}}\right|>s\right\} \subset E_{4} \cup E_{5} \cup E_{6} .
$$

$\forall \vartheta>0$, by Lemma 3.4, there exists $k(\vartheta)$ such that

$$
\operatorname{meas}\left(E_{5}\right) \leq \frac{\vartheta}{3} \text { and meas }\left(E_{6}\right) \leq \frac{\vartheta}{3} .
$$

Using (3.37)-(3.40), we get

$$
\text { meas }\left(\left\{\left|\frac{\partial}{\partial x_{i}} T_{k}\left(u_{n}\right)-\frac{\partial}{\partial x_{i}} T_{k}\left(u_{m}\right)\right|>s\right\}\right) \leq \vartheta,
$$

for all $n, m \geq n_{1}(s, \vartheta)$. Therefore, $\frac{\partial T_{k}\left(u_{\epsilon}\right)}{\partial x_{i}}$ converges in measure to $\frac{\partial T_{k}(u)}{\partial x_{i}}$. Using lemmas 3.5 and 3.10, we deduce that $\frac{\partial T_{k}\left(u_{\epsilon}\right)}{\partial x_{i}}$ converges to $\frac{\partial T_{k}(u)}{\partial x_{i}}$ in $L^{1}(\Omega)$. So, after passing to a suitable subsequence of $\left(\frac{\partial T_{k}\left(u_{\epsilon}\right)}{\partial x_{i}}\right)_{\epsilon>0}$, we can assume that $\frac{\partial T_{k}\left(u_{\epsilon}\right)}{\partial x_{i}}$ converges to $\frac{\partial T_{k}(u)}{\partial x_{i}}$ a.e. in $\Omega$. By the continuity of $a_{i}(x,$.$) , we deduce that a_{i}\left(x, \frac{\partial T_{k}\left(u_{\epsilon}\right)}{\partial x_{i}}\right)$ converges to $a_{i}\left(x, \frac{\partial T_{k}(u)}{\partial x_{i}}\right)$ a.e. in $\Omega$. As $\Omega$ is bounded, this convergence is in measure. Using lemmas 3.10 and 3.12, we deduce that for all $k>0, i=1, \ldots, N$, $a_{i}\left(x, \frac{\partial}{\partial x_{i}} T_{k}\left(u_{\epsilon}\right)\right)$ converges to $a_{i}\left(x, \frac{\partial}{\partial x_{i}} T_{k}(u)\right)$ in $L^{1}(\Omega)$ strongly and $a_{i}\left(x, \frac{\partial}{\partial x_{i}} T_{k}\left(u_{\epsilon}\right)\right)$ converges to $\chi_{k} \in$ $L^{p_{i}^{\prime}(.)}(\Omega)$ weakly in $L^{p_{i}^{\prime}(.)}(\Omega)$. Since each of the convergences implies the weak $L^{1}$-convergence, $\chi_{k}$ can be identified with $a_{i}\left(x, \frac{\partial}{\partial x_{i}} T_{k}(u)\right)$; thus, $a_{i}\left(x, \frac{\partial}{\partial x_{i}} T_{k}(u)\right) \in L^{p_{i}^{\prime}(.)}(\Omega)$

Lemma 3.13. (Lebesgue generalized convergence theorem) Let $\left(f_{n}\right)_{n \in \mathbb{N}}$ be a sequence of measurable functions and $f$ a measurable function such that $f_{n} \rightarrow f$ a.e. in $\Omega$. 
Let $\left(g_{n}\right)_{n \in \mathbb{N}} \subset L^{1}(\Omega)$ be such that $\left|f_{n}\right| \leq g_{n}$ a.e. in $\Omega$ and $g_{n} \rightarrow g$ in $L^{1}(\Omega)$.Then,

$$
\int_{\Omega} f_{n} d x \rightarrow \int_{\Omega} f d x
$$

Proposition 3.3. For any $k>0$ and any $i=1, \ldots, N$, as $\epsilon$ tends to 0 , we have

(i) $\frac{\partial T_{k}\left(u_{\epsilon}\right)}{\partial x_{i}} \rightarrow \frac{\partial T_{k}(u)}{\partial x_{i}}$ a.e. in $\Omega$,

(ii) $a_{i}\left(x, \frac{\partial T_{k}\left(u_{\epsilon}\right)}{\partial x_{i}}\right) \frac{\partial T_{k}\left(u_{\epsilon}\right)}{\partial x_{i}} \rightarrow a_{i}\left(x, \frac{\partial T_{k}(u)}{\partial x_{i}}\right) \frac{\partial T_{k}(u)}{\partial x_{i}}$ a.e. in $\Omega$ and strongly in $L^{1}(\Omega)$,

(iii) $\frac{\partial T_{k}\left(u_{\epsilon}\right)}{\partial x_{i}} \rightarrow \frac{\partial T_{k}(u)}{\partial x_{i}}$ strongly in $L^{p_{i}(x)}(\Omega)$.

Proof. We must show that

$$
\lim _{\epsilon \rightarrow 0} \sum_{i=1}^{N} \int_{\Omega}\left(a_{i}\left(x, \frac{\partial T_{k}\left(u_{\epsilon}\right)}{\partial x_{i}}\right)-a_{i}\left(x, \frac{\partial T_{k}(u)}{\partial x_{i}}\right)\right)\left(\frac{\partial T_{k}\left(u_{\epsilon}\right)}{\partial x_{i}}-\frac{\partial T_{k}(u)}{\partial x_{i}}\right) d x=0 .
$$

Indeed, if the above equality yields, we can assert that for all $i=1, \ldots, N$,

$$
\lim _{\epsilon \rightarrow 0} \int_{\Omega}\left(a_{i}\left(x, \frac{\partial T_{k}\left(u_{\epsilon}\right)}{\partial x_{i}}\right)-a_{i}\left(x, \frac{\partial T_{k}(u)}{\partial x_{i}}\right)\right)\left(\frac{\partial T_{k}\left(u_{\epsilon}\right)}{\partial x_{i}}-\frac{\partial T_{k}(u)}{\partial x_{i}}\right) d x=0 .
$$

Let $i$ be fixed; since

$$
g_{\epsilon}^{i}(.):=\left(a_{i}\left(., \frac{\partial T_{k}\left(u_{\epsilon}\right)}{\partial x_{i}}\right)-a_{i}\left(., \frac{\partial T_{k}(u)}{\partial x_{i}}\right)\right)\left(\frac{\partial T_{k}\left(u_{\epsilon}\right)}{\partial x_{i}}-\frac{\partial T_{k}(u)}{\partial x_{i}}\right) \geq 0
$$

up to a subsequence, we have $g_{\epsilon}^{i}(.) \rightarrow 0$ a.e. in $\Omega$.

This implies that, there exists $Z \subset \Omega$ such that meas $(Z)=0$ and $g_{\epsilon}^{i}(.) \rightarrow 0$ a.e. in $\Omega \backslash Z$.

Let $x \in \Omega \backslash Z$. Using the assumptions $\left(H_{2}\right)$ and $\left(H_{4}\right)$, it follows that the sequence $\left(\frac{\partial T_{k}\left(u_{\epsilon}(x)\right)}{\partial x_{i}}\right)_{\epsilon>0}$ is bounded in $\mathbb{R}^{N}$. So, we can extract a subsequence which converges to some $\tilde{\xi}$ in $\mathbb{R}^{N}$. Passing to the limit in the expression of $g_{\epsilon}^{i}(x)$, we get

$$
0=\left(a_{i}(x, \tilde{\xi})-a_{i}\left(x, \frac{\partial T_{k}(u(x))}{\partial x_{i}}\right)\right)\left(\tilde{\xi}-\frac{\partial T_{k}(u(x))}{\partial x_{i}}\right)
$$

This yields $\tilde{\xi}=\frac{\partial T_{k}(u(x))}{\partial x_{i}}, \forall x \in \Omega \backslash Z$. As the limit does not depend on the subsequence, the whole sequence $\left(\frac{\partial T_{k}\left(u_{\epsilon}(x)\right)}{\partial x_{i}}\right)_{\epsilon>0}$ converges to $\tilde{\xi}$ in $\mathbb{R}^{N}$. This means that $\frac{\partial T_{k}\left(u_{\epsilon}\right)}{\partial x_{i}} \rightarrow \frac{\partial T_{k}(u)}{\partial x_{i}}$ a.e. in $\Omega$.

We have to show (3.42). The proof consists in three steps.

Step 1. We prove that for every function $h \in W^{1, \infty}(\mathbb{R}), h>0$ with compact support,

$$
\left\{\begin{array}{l}
\limsup _{\epsilon \rightarrow 0} \sum_{i=1}^{N} \int_{\Omega}\left(a_{i}\left(x, \frac{\partial u_{\epsilon}}{\partial x_{i}}\right) \frac{\partial\left[h\left(u_{\epsilon}\right)\left(T_{k}\left(u_{\epsilon}\right)-T_{k}(u)\right)\right]}{\partial x_{i}}\right) d x \\
+\limsup _{\epsilon \rightarrow 0} \sum_{i=1}^{N} \int_{\tilde{\Omega} \backslash \Omega}\left(\frac{1}{\epsilon^{p_{i}(x)}}\left|\frac{\partial u_{\epsilon}}{\partial x_{i}}\right|^{p_{i}(x)-2} \frac{\partial u_{\epsilon}}{\partial x_{i}} \frac{\partial\left[h\left(u_{\epsilon}\right)\left(T_{k}\left(u_{\epsilon}\right)-T_{k}(u)\right)\right]}{\partial x_{i}}\right) d x \leq 0 .
\end{array}\right.
$$


Taking $\tilde{\xi}=h\left(u_{\epsilon}\right)\left(T_{k}\left(u_{\epsilon}\right)-T_{k}(u)\right)$ as test function in (3.19), we have

$$
\left\{\begin{array}{l}
\sum_{i=1}^{N} \int_{\Omega}\left(a_{i}\left(x, \frac{\partial}{\partial x_{i}} u_{\epsilon}\right) \frac{\partial}{\partial x_{i}}\left[h\left(u_{\epsilon}\right)\left(T_{k}\left(u_{\epsilon}\right)-T_{k}(u)\right)\right]\right) d x \\
+\sum_{i=1}^{N} \int_{\tilde{\Omega} \backslash \Omega}\left(\frac{1}{\epsilon^{p_{i}(x)}}\left|\frac{\partial}{\partial x_{i}} u_{\epsilon}\right|^{p_{i}(x)-2} \frac{\partial}{\partial x_{i}} u_{\epsilon} \frac{\partial}{\partial x_{i}}\left[h\left(u_{\epsilon}\right)\left(T_{k}\left(u_{\epsilon}\right)-T_{k}(u)\right)\right]\right) d x \\
+\int_{\Omega} b\left(u_{\epsilon}\right)\left[h\left(u_{\epsilon}\right)\left(T_{k}\left(u_{\epsilon}\right)-T_{k}(u)\right)\right] d x=\int_{\Omega} f_{\epsilon}\left[h\left(u_{\epsilon}\right)\left(T_{k}\left(u_{\epsilon}\right)-T_{k}(u)\right)\right] d x \\
+\int_{\tilde{\Gamma}_{N e}}\left(\tilde{d}-\tilde{\rho}\left(u_{\epsilon}\right)\left[h\left(u_{\epsilon}\right)\left(T_{k}\left(u_{\epsilon}\right)-T_{k}(u)\right)\right] d \sigma .\right.
\end{array}\right.
$$

Note that

$$
\begin{aligned}
\int_{\Omega} b\left(u_{\epsilon}\right)\left[h\left(u_{\epsilon}\right)\left(T_{k}\left(u_{\epsilon}\right)-T_{k}(u)\right)\right] d x & =\int_{\Omega}\left[b\left(u_{\epsilon}\right)-b(u)\right] h\left(u_{\epsilon}\right)\left(T_{k}\left(u_{\epsilon}\right)-T_{k}(u)\right) d x \\
& +\int_{\Omega} b(u) h\left(u_{\epsilon}\right)\left(T_{k}\left(u_{\epsilon}\right)-T_{k}(u)\right) d x
\end{aligned}
$$

Since

$$
\int_{\Omega}\left[b\left(u_{\epsilon}\right)-b(u)\right] h\left(u_{\epsilon}\right)\left(T_{k}\left(u_{\epsilon}\right)-T_{k}(u)\right) d x \geq 0
$$

and

$$
\lim _{\epsilon \rightarrow 0} \int_{\Omega} b(u) h\left(u_{\epsilon}\right)\left(T_{k}\left(u_{\epsilon}\right)-T_{k}(u)\right) d x=0
$$

we get

$$
\limsup _{\epsilon \rightarrow 0} \int_{\Omega} b\left(u_{\epsilon}\right)\left[h\left(u_{\epsilon}\right)\left(T_{k}\left(u_{\epsilon}\right)-T_{k}(u)\right)\right] d x \geq 0 .
$$

We also have

$$
\begin{aligned}
\int_{\tilde{\Gamma}_{N e}} \tilde{\rho}\left(u_{\epsilon}\right)\left[h\left(u_{\epsilon}\right)\left(T_{k}\left(u_{\epsilon}\right)-T_{k}(u)\right)\right] d x & =\int_{\tilde{\Gamma}_{N e}}\left[\tilde{\rho}\left(u_{\epsilon}\right)-\tilde{\rho}(u)\right] h\left(u_{\epsilon}\right)\left(T_{k}\left(u_{\epsilon}\right)-T_{k}(u)\right) d x \\
& +\int_{\tilde{\Gamma}_{N e}} \tilde{\rho}(u) h\left(u_{\epsilon}\right)\left(T_{k}\left(u_{\epsilon}\right)-T_{k}(u)\right) d x
\end{aligned}
$$

Since

and

$$
\int_{\tilde{\Gamma}_{N e}}\left[\tilde{\rho}\left(u_{\epsilon}\right)-\tilde{\rho}(u)\right] h\left(u_{\epsilon}\right)\left(T_{k}\left(u_{\epsilon}\right)-T_{k}(u)\right) d x \geq 0
$$

$$
\lim _{\epsilon \rightarrow 0} \int_{\tilde{\Gamma}_{N e}} \tilde{\rho}(u) h\left(u_{\epsilon}\right)\left(T_{k}\left(u_{\epsilon}\right)-T_{k}(u)\right) d x=0
$$

we get

$$
\limsup _{\epsilon \rightarrow 0} \int_{\tilde{\Gamma}_{N e}} \tilde{\rho}\left(u_{\epsilon}\right) h\left(u_{\epsilon}\right)\left(T_{k}\left(u_{\epsilon}\right)-T_{k}(u)\right) d x \geq 0
$$

By Lemma 3.13, we have

$$
\lim _{\epsilon \rightarrow 0} \int_{\Omega} f_{\epsilon} h\left(u_{\epsilon}\right)\left(T_{k}\left(u_{\epsilon}\right)-T_{k}(u)\right) d x=0
$$

and

$$
\lim _{\epsilon \rightarrow 0} \int_{\tilde{\Gamma}_{N e}} \tilde{d} h\left(u_{\epsilon}\right)\left(T_{k}\left(u_{\epsilon}\right)-T_{k}(u)\right) d x=0 .
$$

Letting $\epsilon$ goes to zero in (3.44) by using (3.45)-(3.48) we get (3.43). 
Step 2. We prove that

$$
\limsup _{l \rightarrow \infty} \limsup _{\epsilon \rightarrow 0} \sum_{i=1}^{N} \int_{[l<|u|<l+1]} a_{i}\left(x, \frac{\partial u_{\epsilon}}{\partial x_{i}}\right) \frac{\partial u_{\epsilon}}{\partial x_{i}} \leq 0 .
$$

Taking $w_{l}\left(u_{\epsilon}\right)$ as test function in (3.19), where $w_{l}(r)=T_{1}\left(r-T_{l}(r)\right)$, we get

$$
\left\{\begin{array}{l}
\sum_{i=1}^{N} \int_{\Omega \cap\left[l<\left|u_{\epsilon}\right|<l+1\right]}\left(a_{i}\left(x, \frac{\partial}{\partial x_{i}} u_{\epsilon}\right) \frac{\partial}{\partial x_{i}} u_{\epsilon}\right) d x \\
+\sum_{i=1}^{N} \int_{\tilde{\Omega} \backslash \Omega \cap\left[l<\left|u_{\epsilon}\right|<l+1\right]}\left(\frac{1}{\epsilon^{p_{i}(x)}}\left|\frac{\partial}{\partial x_{i}} u_{\epsilon}\right|^{p_{i}(x)}\right) d x \\
+\int_{\Omega} b\left(u_{\epsilon}\right)\left(T_{1}\left(u_{\epsilon}-T_{l}(u)\right) d x=\int_{\Omega} f_{\epsilon}\left(T_{1}\left(u_{\epsilon}-T_{l}(u)\right) d x\right.\right. \\
+\int_{\tilde{\Gamma}_{N e}}\left(\tilde{d}-\tilde{\rho}\left(u_{\epsilon}\right)\left(T_{1}\left(u_{\epsilon}-T_{l}(u)\right) d \sigma .\right.\right.
\end{array}\right.
$$

In (3.50), the integrals $\sum_{i=1}^{N} \int_{\tilde{\Omega} \backslash \Omega \cap\left[l<\left|u_{\epsilon}\right|<l+1\right]}\left(\frac{1}{\epsilon^{p_{i}(x)}}\left|\frac{\partial}{\partial x_{i}} u_{\epsilon}\right|^{p_{i}(x)}\right) d x$,

$\int_{\Omega} b\left(u_{\epsilon}\right)\left(T_{1}\left(u_{\epsilon}-T_{l}(u)\right) d x\right.$ and $\int_{\tilde{\Gamma}_{N e}} \tilde{\rho}\left(u_{\epsilon}\right) T_{1}\left(u_{\epsilon}-T_{l}(u)\right) d \sigma$ are non-negative and it is easy to see that

$$
\limsup _{l \rightarrow \infty} \limsup _{\epsilon \rightarrow 0} \int_{\Omega} f_{\epsilon}\left(T_{1}\left(u_{\epsilon}-T_{l}(u)\right) d x=0=\limsup _{l \rightarrow \infty} \limsup _{\epsilon \rightarrow 0} \int_{\tilde{\Gamma}_{N e}} \tilde{d}\left(T_{1}\left(u_{\epsilon}-T_{l}(u)\right) d \sigma .\right.\right.
$$

Hence, (3.49) follows by passing to the limit in (3.50).

Step 3. We prove that for every $k>0$,

$$
\limsup _{\epsilon \rightarrow 0} \sum_{i=1}^{N} \int_{\Omega}\left(a_{i}\left(x, \frac{\partial}{\partial x_{i}} u_{\epsilon}\right) \cdot \frac{\partial}{\partial x_{i}}\left(T_{k}\left(u_{\epsilon}\right)-T_{k}(u)\right)\right) d x \leq 0 .
$$

For all $l>0$, we define the function $h_{l}$ by $h_{l}(r)=\inf \left\{1,(l+1-|r|)^{+}\right\}$.

For all $l>k$, we have

$$
\left\{\begin{array}{l}
\sum_{i=1}^{N} \int_{\Omega}\left(a_{i}\left(x, \frac{\partial}{\partial x_{i}} u_{\epsilon}\right) \frac{\partial}{\partial x_{i}}\left[h_{l}\left(u_{\epsilon}\right)\left(T_{k}\left(u_{\epsilon}\right)-T_{k}(u)\right)\right]\right) d x \\
=\sum_{i=1}^{N} \int_{\left[\left|u_{\epsilon}\right| \leq k\right]}\left(h_{l}\left(u_{\epsilon}\right) a_{i}\left(x, \frac{\partial}{\partial x_{i}} u_{\epsilon}\right) \frac{\partial}{\partial x_{i}}\left(T_{k}\left(u_{\epsilon}\right)-T_{k}(u)\right)\right) d x \\
+\sum_{i=1}^{N} \int_{\left[\left|u_{\epsilon}\right|>k\right]}\left(h_{l}\left(u_{\epsilon}\right) a_{i}\left(x, \frac{\partial}{\partial x_{i}} u_{\epsilon}\right) \frac{\partial}{\partial x_{i}}\left(-T_{k}(u)\right)\right) d x \\
+\sum_{i=1}^{N} \int_{\Omega}\left(h_{l}^{\prime}\left(u_{\epsilon}\right)\left(T_{k}\left(u_{\epsilon}\right)-T_{k}(u)\right) a_{i}\left(x, \frac{\partial}{\partial x_{i}} u_{\epsilon}\right) \frac{\partial u_{\epsilon}}{\partial x_{i}}\right) d x= \\
E_{1}+E_{2}+E_{3} .
\end{array}\right.
$$

Since $l>k$, on the set $\left[\left|u_{\epsilon}\right| \leq k\right]$ we have $h_{l}\left(u_{\epsilon}\right)=1$ so that we can write $\left(E_{1}\right)$ as

$$
\left(E_{1}\right)=\sum_{i=1}^{N} \int_{\Omega}\left(a_{i}\left(x, \frac{\partial}{\partial x_{i}} T_{k}\left(u_{\epsilon}\right)\right) \frac{\partial}{\partial x_{i}}\left(T_{k}\left(u_{\epsilon}\right)-T_{k}(u)\right)\right) d x .
$$

Hence, we obtain

$$
\limsup _{\epsilon \rightarrow 0}\left(E_{1}\right)=\limsup _{\epsilon \rightarrow 0} \sum_{i=1}^{N} \int_{\Omega}\left(a_{i}\left(x, \frac{\partial}{\partial x_{i}} T_{k}\left(u_{\epsilon}\right)\right) \frac{\partial}{\partial x_{i}}\left(T_{k}\left(u_{\epsilon}\right)-T_{k}(u)\right)\right) d x .
$$


Let us write the term $\left(E_{2}\right)$ as

$$
\left(E_{2}\right)=-\sum_{i=1}^{N} \int_{\left[\left|u_{\epsilon}\right|>k\right]}\left(h_{l}\left(u_{\epsilon}\right) a_{i}\left(x, \frac{\partial}{\partial x_{i}} T_{l+1}\left(u_{\epsilon}\right)\right) \frac{\partial}{\partial x_{i}} T_{k}(u)\right) d x .
$$

Using Lebesgue dominated convergence theorem, we get

$$
\limsup _{\epsilon \rightarrow 0}\left(E_{2}\right)=-\sum_{i=1}^{N} \int_{[|u|>k]}\left(h_{l}(u) a_{i}\left(x, \frac{\partial}{\partial x_{i}} T_{l+1}(u)\right) \frac{\partial}{\partial x_{i}} T_{k}(u)\right) d x=0 .
$$

For the term $\left(E_{3}\right)$, we have

$$
\left\{\begin{array}{l}
\left(E_{3}\right) \leq\left|\sum_{i=1}^{N} \int_{\Omega}\left(h_{l}^{\prime}\left(u_{\epsilon}\right)\left(T_{k}\left(u_{\epsilon}\right)-T_{k}(u)\right) a_{i}\left(x, \frac{\partial}{\partial x_{i}} u_{\epsilon}\right) \frac{\partial u_{\epsilon}}{\partial x_{i}}\right) d x\right| \\
\leq 2 k \sum_{i=1}^{N} \int_{[l<|u|<l+1]}\left(a_{i}\left(x, \frac{\partial}{\partial x_{i}} u_{\epsilon}\right) \frac{\partial u_{\epsilon}}{\partial x_{i}}\right) d x
\end{array}\right.
$$

Using the result of the Step 2 we deduce that

$$
\limsup _{l \rightarrow \infty} \limsup _{\epsilon \rightarrow 0}\left(E_{3}\right) \leq 0
$$

Applying (3.43) with $h$ replaced by $h_{l}, l>k$ we get

$$
\left\{\begin{array}{l}
\limsup _{\epsilon \rightarrow 0} \sum_{i=1}^{N} \int_{\Omega}\left(a_{i}\left(x, \frac{\partial T_{k}\left(u_{\epsilon}\right)}{\partial x_{i}}\right) \frac{\partial\left(T_{k}\left(u_{\epsilon}\right)-T_{k}(u)\right)}{\partial x_{i}}\right) d x \\
+\limsup _{\epsilon \rightarrow 0} \sum_{i=1}^{N} \int_{\tilde{\Omega} \backslash \Omega}\left(\frac{1}{\epsilon^{p_{i}(x)}}\left|\frac{\partial u_{\epsilon}}{\partial x_{i}}\right|^{p_{i}(x)-2} \frac{\partial u_{\epsilon}}{\partial x_{i}} \frac{\partial\left[h_{l}\left(u_{\epsilon}\right)\left(T_{k}\left(u_{\epsilon}\right)-T_{k}(u)\right)\right]}{\partial x_{i}}\right) d x \leq-\limsup _{\epsilon \rightarrow 0} E_{3} .
\end{array}\right.
$$

This last inequality imply that

$$
\left\{\begin{array}{l}
\limsup _{\epsilon \rightarrow 0} \sum_{i=1}^{N} \int_{\Omega}\left(a_{i}\left(x, \frac{\partial T_{k}\left(u_{\epsilon}\right)}{\partial x_{i}}\right) \frac{\partial\left(T_{k}\left(u_{\epsilon}\right)-T_{k}(u)\right)}{\partial x_{i}}\right) d x \\
+\limsup _{\epsilon \rightarrow 0} \sum_{i=1}^{N} \int_{\tilde{\Omega} \backslash \Omega}\left(\frac{1}{\epsilon^{p_{i}(x)}}\left|\frac{\partial T_{k}\left(u_{\epsilon}\right)}{\partial x_{i}}\right|^{p_{i}(x)-2} \frac{\partial T_{k}\left(u_{\epsilon}\right)}{\partial x_{i}} \frac{\partial\left[h_{l}\left(u_{\epsilon}\right)\left(T_{k}\left(u_{\epsilon}\right)-T_{k}(u)\right)\right]}{\partial x_{i}}\right) d x \\
\leq-\limsup _{\epsilon \rightarrow 0} E_{3} .
\end{array}\right.
$$

Note that

$$
\left\{\begin{array}{l}
\limsup _{\epsilon \rightarrow 0} \sum_{i=1}^{N} \int_{\tilde{\Omega} \backslash \Omega}\left(\frac{1}{\epsilon^{p_{i}(x)}}\left|\frac{\partial T_{k}\left(u_{\epsilon}\right)}{\partial x_{i}}\right|^{p_{i}(x)-2} \frac{\partial T_{k}\left(u_{\epsilon}\right)}{\partial x_{i}} \frac{\partial\left[h_{l}\left(u_{\epsilon}\right)\left(T_{k}\left(u_{\epsilon}\right)-T_{k}(u)\right)\right]}{\partial x_{i}}\right) d x \\
=\limsup _{\epsilon \rightarrow 0} \sum_{i=1}^{N} \int_{\tilde{\Omega} \backslash \Omega}\left(\frac{1}{\epsilon^{p_{i}(x)}}\left|\frac{\partial T_{k}\left(u_{\epsilon}\right)}{\partial x_{i}}\right|^{p_{i}(x)-2} \frac{\partial T_{k}\left(u_{\epsilon}\right)}{\partial x_{i}} \frac{\partial\left(T_{k}\left(u_{\epsilon}\right)-T_{k}(u)\right)}{\partial x_{i}}\right) d x \\
=\limsup _{\epsilon \rightarrow 0} \sum_{i=1}^{N} \int_{\tilde{\Omega} \backslash \Omega}\left(\frac{1}{\epsilon^{p_{i}(x)}}\left|\frac{\partial T_{k}\left(u_{\epsilon}\right)}{\partial x_{i}}\right|^{p_{i}(x)}\right) d x \geq 0 ;
\end{array}\right.
$$

since $\forall i=1, \ldots, N, \frac{\partial T_{k}(u)}{\partial x_{i}}=0$ on $\tilde{\Omega} \backslash \Omega$, where in the first Step we have used the fact that $h_{l}\left(u_{\epsilon}\right)=1$ on $\left[\left|u_{\epsilon}\right| \leq k\right]$. Hence from (3.52), we get

$$
\limsup _{\epsilon \rightarrow 0} \sum_{i=1}^{N} \int_{\Omega}\left(a_{i}\left(x, \frac{\partial T_{k}\left(u_{\epsilon}\right)}{\partial x_{i}}\right) \frac{\partial\left(T_{k}\left(u_{\epsilon}\right)-T_{k}(u)\right)}{\partial x_{i}}\right) d x \leq-\limsup _{\epsilon \rightarrow 0} E_{3} .
$$


By letting $l$ goes to $\infty$ in (3.53), the inequality (3.51) follows.

Thanks to (3.51), we deduce that for all $k>0$,

$$
\lim _{\epsilon \rightarrow 0} \sum_{i=1}^{N} \int_{\Omega}\left(a_{i}\left(x, \frac{\partial T_{k}\left(u_{\epsilon}\right)}{\partial x_{i}}\right)-a_{i}\left(x, \frac{\partial T_{k}(u)}{\partial x_{i}}\right)\right)\left(\frac{\partial T_{k}\left(u_{\epsilon}\right)}{\partial x_{i}}-\frac{\partial T_{k}(u)}{\partial x_{i}}\right) d x=0 .
$$

(ii) The continuity of $a_{i}(x, \xi)$ for any $i=1, \ldots, N$ with respect to $\tilde{\xi} \in \mathbb{R}^{N}$ gives us

$$
a_{i}\left(x, \frac{\partial T_{k}\left(u_{\epsilon}\right)}{\partial x_{i}}\right) \rightarrow a_{i}\left(x, \frac{\partial T_{k}(u)}{\partial x_{i}}\right) \text { a.e. in } \Omega,
$$

for all $i=1, \ldots, N$. Then, we obtain for all $i=1, \ldots, N$,

$$
a_{i}\left(x, \frac{\partial T_{k}\left(u_{\epsilon}\right)}{\partial x_{i}}\right) \frac{\partial T_{k}\left(u_{\epsilon}\right)}{\partial x_{i}} \rightarrow a_{i}\left(x, \frac{\partial T_{k}(u)}{\partial x_{i}}\right) \frac{\partial T_{k}(u)}{\partial x_{i}} \text { a.e. in } \Omega .
$$

For $i$ fixed, setting $y_{\epsilon}^{i}=a_{i}\left(x, \frac{\partial T_{k}\left(u_{\epsilon}\right)}{\partial x_{i}}\right) \frac{\partial T_{k}\left(u_{\epsilon}\right)}{\partial x_{i}}$ and $y^{i}=a_{i}\left(x, \frac{\partial T_{k}(u)}{\partial x_{i}}\right) \frac{\partial T_{k}(u)}{\partial x_{i}}$, we have $y_{\epsilon}^{i} \geq 0, y_{\epsilon}^{i} \rightarrow y^{i}$ a.e. in $\Omega$, $y^{i} \in L^{1}(\Omega), \int_{\Omega} y_{\epsilon}^{i} d x \rightarrow \int_{\Omega} y^{i} d x$. Since $\int_{\Omega}\left|y_{\epsilon}^{i}-y^{i}\right| d x=2 \int_{\Omega}\left(y^{i}-y_{\epsilon}^{i}\right)^{+} d x+\int_{\Omega}\left(y_{\epsilon}^{i}-y^{i}\right) d x$, and $\left(y^{i}-y_{\epsilon}^{i}\right)^{+} \leq y^{i}$, it follow by the Lebesgue dominated convergence theorem that $\lim _{\epsilon \rightarrow 0} \int_{\Omega}\left|y_{\epsilon}^{i}-y^{i}\right| d x=0$, which means that $a_{i}\left(x, \frac{\partial T_{k}\left(u_{\epsilon}\right)}{\partial x_{i}}\right) \frac{\partial T_{k}\left(u_{\epsilon}\right)}{\partial x_{i}} \rightarrow a_{i}\left(x, \frac{\partial T_{k}(u)}{\partial x_{i}}\right) \frac{\partial T_{k}(u)}{\partial x_{i}}$ strongly in $L^{1}(\Omega) \square$

(iii) By $\left(H_{4}\right)$, for all $i=1, \ldots, N$, one has

$$
\left|T_{k}\left(u_{\epsilon}\right)\right|^{p_{i}(x)} \leq a_{i}\left(x, \frac{\partial T_{k}\left(u_{\epsilon}\right)}{\partial x_{i}}\right) \frac{\partial T_{k}\left(u_{\epsilon}\right)}{\partial x_{i}} .
$$

Using the $L^{1}$-convergence of $(i i)$ and the generalized dominated convergence Theorem, the result of (iii) follows

\section{EXISTENCE AND UNIQUENESS OF ENTROPY SOlUtion}

We are now able to prove Theorem 2.4.

Proof of Theorem 2.4. Thanks to the Proposition 3.2 and as $\forall k>0, \forall i=1, \ldots, N, \frac{\partial T_{k}(u)}{\partial x_{i}}=0$ in $L^{p_{i}^{-}}(\tilde{\Omega} \backslash \Omega)$, then $\forall k>0, T_{k}(u)=$ constant a.e. on $\tilde{\Omega} \backslash \Omega$. Hence, we conclude that $u \in \mathcal{T}_{N e}^{1, \vec{p}(.)}(\Omega)$.

We already state that $b(u) \in L^{1}(\Omega)$.

To show that $u$ is an entropy solution of $P(\rho, f, d)$, we only have to prove the inequality in (2.9).

Let $\varphi \in W_{D}^{1, \vec{p}(.)}(\Omega) \cap L^{\infty}(\Omega)$. We consider the function $\varphi_{1} \in W_{D}^{1, \vec{p}(.)}(\tilde{\Omega}) \cap L^{\infty}(\Omega)$ such that

$$
\varphi_{1}=\varphi \chi_{\Omega}+\varphi_{N} \chi_{\tilde{\Omega} \backslash \Omega}
$$

we set $\tilde{\xi}=T_{k}\left(u_{\epsilon}-\varphi_{1}\right)$ in (3.19) to get

$$
\left\{\begin{array}{l}
\sum_{i=1}^{N} \int_{\Omega}\left(a_{i}\left(x, \frac{\partial}{\partial x_{i}} u_{\epsilon}\right) \cdot \frac{\partial}{\partial x_{i}} T_{k}\left(u_{\epsilon}-\varphi\right)\right) d x \\
+\sum_{i=1}^{N} \int_{\tilde{\Omega} \backslash \Omega}\left(\frac{1}{\epsilon^{p_{i}(x)}}\left|\frac{\partial}{\partial x_{i}} u_{\epsilon}\right|^{p_{i}(x)-2} \frac{\partial}{\partial x_{i}} u_{\epsilon} \cdot \frac{\partial}{\partial x_{i}} T_{k}\left(u_{\epsilon}-\varphi_{N}\right)\right) d x \\
\int_{\Omega} b\left(u_{\epsilon}\right) T_{k}\left(u_{\epsilon}-\varphi\right) d x=\int_{\Omega} f_{\epsilon} \tilde{\xi} d x+\int_{\tilde{\Gamma}_{N e}}\left(\tilde{d}-\tilde{\rho}\left(u_{\epsilon}\right)\right) T_{k}\left(u_{\epsilon}-\varphi_{N}\right) d \sigma .
\end{array}\right.
$$

The following convergence result holds true. 
Lemma 4.1. For any $k>0$, for all $i=1, \ldots, N$, as $\epsilon \rightarrow 0$,

$$
\frac{\partial}{\partial x_{i}} T_{k}\left(u_{\epsilon}-\varphi\right) \rightarrow \frac{\partial}{\partial x_{i}} T_{k}(u-\varphi) \text { strongly in } L^{p_{i}(.)}(\Omega)
$$

Proof. Let $k>0, i=1, \ldots, N$. We have

$$
\begin{aligned}
\int_{\Omega}\left|\frac{\partial}{\partial x_{i}} T_{k}\left(u_{\epsilon}-\varphi\right)-\frac{\partial}{\partial x_{i}} T_{k}(u-\varphi)\right|^{p_{i}(x)} d x & =\int_{\Omega \cap\left[\left|u_{\epsilon}-\varphi\right| \leq k,|u-\varphi| \leq k\right]}\left|\frac{\partial}{\partial x_{i}} u_{\epsilon}-\frac{\partial}{\partial x_{i}} u\right|^{p_{i}(x)} d x \\
& \leq \int_{\Omega \cap\left[\left|u_{\epsilon}\right| \leq l,|u| \leq l\right]}\left|\frac{\partial u_{\epsilon}}{\partial x_{i}}-\frac{\partial u}{\partial x_{i}}\right|^{p_{i}(x)} d x, \text { with } l=k+\|\varphi\|_{\infty} \\
& =\int_{\Omega}\left|\frac{\partial}{\partial x_{i}} T_{l}\left(u_{\epsilon}\right)-\frac{\partial}{\partial x_{i}} T_{l}(u)\right|^{p_{i}(x)} d x \\
& \rightarrow 0 \text { as } \epsilon \rightarrow 0 \text { by Proposition } 3.3-(i i i) .
\end{aligned}
$$

This completes the proof of the Lemma 4.1.

We need to pass to the limit in (4.1) as $\epsilon \rightarrow 0$. We have

$$
\sum_{i=1}^{N} \int_{\Omega}\left(a_{i}\left(x, \frac{\partial}{\partial x_{i}} u_{\epsilon}\right) \frac{\partial}{\partial x_{i}} T_{k}\left(u_{\epsilon}-\varphi\right)\right) d x=\sum_{i=1}^{N} \int_{\Omega}\left(a_{i}\left(x, \frac{\partial T_{l}\left(u_{\epsilon}\right)}{\partial x_{i}}\right) \frac{\partial}{\partial x_{i}} T_{k}\left(u_{\epsilon}-\varphi\right)\right) d x,
$$

with $l=k+\|\varphi\|_{\infty}$, then, by Lemma 3.8-(ii) and Lemma 4.1, we have

$$
\lim _{\epsilon \rightarrow 0} \sum_{i=1}^{N} \int_{\Omega}\left(a_{i}\left(x, \frac{\partial T_{l}\left(u_{\epsilon}\right)}{\partial x_{i}}\right) \frac{\partial}{\partial x_{i}} T_{k}\left(u_{\epsilon}-\varphi\right)\right) d x=\sum_{i=1}^{N} \int_{\Omega}\left(a_{i}\left(x, \frac{\partial T_{l}(u)}{\partial x_{i}}\right) \frac{\partial}{\partial x_{i}} T_{k}(u-\varphi)\right) d x ;
$$

that is

$$
\lim _{\epsilon \rightarrow 0} \sum_{i=1}^{N} \int_{\Omega}\left(a_{i}\left(x, \frac{\partial}{\partial x_{i}} u_{\epsilon}\right) \frac{\partial}{\partial x_{i}} T_{k}\left(u_{\epsilon}-\varphi\right)\right) d x=\sum_{i=1}^{N} \int_{\Omega}\left(a_{i}\left(x, \frac{\partial T_{l}(u)}{\partial x_{i}}\right) \frac{\partial}{\partial x_{i}} T_{k}(u-\varphi)\right) d x .
$$

For the second term in the left hand side of (4.1), we have

$$
\limsup _{\epsilon \rightarrow 0} \sum_{i=1}^{N} \int_{\tilde{\Omega} \backslash \Omega}\left(\frac{1}{\epsilon^{p_{i}(x)}}\left|\frac{\partial}{\partial x_{i}} u_{\epsilon}\right|^{p_{i}(x)-2} \frac{\partial}{\partial x_{i}} u_{\epsilon} \frac{\partial}{\partial x_{i}} T_{k}\left(u_{\epsilon}-\varphi_{N}\right)\right) d x \geq 0 .
$$

Indeed

$$
\left\{\begin{array}{l}
\sum_{i=1}^{N} \int_{\tilde{\Omega} \backslash \Omega}\left(\frac{1}{\epsilon^{p_{i}(x)}}\left|\frac{\partial}{\partial x_{i}} u_{\epsilon}\right|^{p_{i}(x)-2} \frac{\partial}{\partial x_{i}} u_{\epsilon} \cdot \frac{\partial}{\partial x_{i}} T_{k}\left(u_{\epsilon}-\varphi_{N}\right)\right) d x \\
=\sum_{i=1}^{N} \int_{\tilde{\Omega} \backslash \Omega \cap\left[\left|u_{\epsilon}-\varphi\right| \leq k\right]}\left(\frac{1}{\epsilon^{p_{i}(x)}}\left|\frac{\partial}{\partial x_{i}} u_{\epsilon}\right|^{p_{i}(x)-2} \frac{\partial}{\partial x_{i}} u_{\epsilon} \cdot \frac{\partial}{\partial x_{i}}\left(u_{\epsilon}-\varphi_{N}\right)\right) d x \\
\sum_{i=1}^{N} \int_{\tilde{\Omega} \backslash \Omega \cap\left[\left|u_{\epsilon}-\varphi\right| \leq k\right]}\left(\frac{1}{\epsilon^{p_{i}(x)}}\left|\frac{\partial}{\partial x_{i}} u_{\epsilon}\right|^{p_{i}(x)}\right) d x \geq 0 .
\end{array}\right.
$$

Hence, we get (4.3).

Let us examine the last term in the left hand side of (4.1).

we have

$$
\left.\int_{\Omega} b\left(u_{\epsilon}\right) T_{k}\left(u_{\epsilon}-\varphi\right) d x=\int_{\Omega} b\left(u_{\epsilon}\right)-b(\varphi)\right) T_{k}\left(u_{\epsilon}-\varphi\right) d x+\int_{\Omega} b(\varphi) T_{k}\left(u_{\epsilon}-\varphi\right) d x .
$$

As $b$ is non-decreasing,

$$
\left(b\left(u_{\epsilon}\right)-b(\varphi)\right) T_{k}\left(u_{\epsilon}-\varphi\right) \geq 0 \text { a.e. in } \Omega
$$


and we get by Fatou's Lemma that

$$
\liminf _{\epsilon \rightarrow 0} \int_{\Omega}\left(b\left(u_{\epsilon}\right)-b(\varphi)\right) T_{k}\left(u_{\epsilon}-\varphi\right) d x \geq \int_{\Omega}(b(u)-b(\varphi)) T_{k}(u-\varphi) d x .
$$

As $\varphi \in L^{\infty}(\Omega)$, we obtain $b(\varphi) \in L^{\infty}(\Omega)$ and so $b(\varphi) \in L^{1}(\Omega)$ (as $\Omega$ is bounded) and by Lebesgue dominated convergence theorem, we deduce that

$$
\lim _{\epsilon \rightarrow 0} \int_{\Omega} b(\varphi) T_{k}\left(u_{\epsilon}-\varphi\right) d x=\int_{\Omega} b(\varphi) T_{k}(u-\varphi) d x .
$$

Consequently,

$$
\limsup _{\epsilon \rightarrow 0} \int_{\Omega} b\left(u_{\epsilon}\right) T_{k}\left(u_{\epsilon}-\varphi\right) d x \geq \int_{\Omega} b(u) T_{k}(u-\varphi) d x .
$$

It is easy to see by the Lebesgue generalized convergence theorem that

$$
\left\{\begin{array}{l}
\lim _{\epsilon \rightarrow 0} \int_{\Omega} f_{\epsilon} T_{k}\left(u_{\epsilon}-\varphi\right) d x=\int_{\Omega} f T_{k}(u-\varphi) d x \\
\lim _{\epsilon \rightarrow 0} \int_{\tilde{\Gamma}_{N e}} \tilde{d}_{\epsilon} T_{k}\left(u_{\epsilon}-\varphi_{N}\right) d \sigma=\int_{\Omega} \tilde{d} T_{k}\left(u-\varphi_{N}\right) d \sigma .
\end{array}\right.
$$

We know that $\forall k>0, T_{k}(u)=$ constant on $\tilde{\Omega} \backslash \Omega$, then, it yields that $u=$ constant on $\tilde{\Omega} \backslash \Omega$. So, one has

$$
\lim _{\epsilon \rightarrow 0} \int_{\tilde{\Gamma}_{N e}} \tilde{d}_{\epsilon} T_{k}\left(u_{\epsilon}-\varphi\right) d x=d T_{k}\left(u_{N}-\varphi_{N}\right) .
$$

At last, we have

$$
\int_{\tilde{\Gamma}_{N e}} \tilde{\rho}\left(u_{\epsilon}\right) T_{k}\left(u_{\epsilon}-\varphi_{N}\right) d \sigma=\int_{\tilde{\Gamma}_{N e}}\left(\tilde{\rho}\left(u_{\epsilon}\right)-\tilde{\rho}\left(\varphi_{N}\right)\right) T_{k}\left(u_{\epsilon}-\varphi_{N}\right) d \sigma+\int_{\tilde{\Gamma}_{N e}} \tilde{\rho}\left(\varphi_{N}\right) T_{k}\left(u_{\epsilon}-\varphi_{N}\right) d \sigma .
$$

As $\tilde{\rho}$ is non-decreasing,

$$
\left(\tilde{\rho}\left(u_{\epsilon}\right)-\tilde{\rho}\left(\varphi_{N}\right)\right) T_{k}\left(u_{\epsilon}-\varphi_{N}\right) \geq 0 \text { a.e. on } \tilde{\Gamma}_{N e}
$$

and we get by Fatou's Lemma that

$$
\begin{aligned}
\liminf _{\epsilon \rightarrow 0} \int_{\tilde{\Gamma}_{N e}}\left(\tilde{\rho}\left(u_{\epsilon}\right)-\tilde{\rho}\left(\varphi_{N}\right)\right) T_{k}\left(u_{\epsilon}-\varphi_{N}\right) d \sigma & \geq \int_{\tilde{\Gamma}_{N e}}\left(\tilde{\rho}\left(u_{N}\right)-\tilde{\rho}\left(\varphi_{N}\right)\right) T_{k}\left(u_{N}-\varphi_{N}\right) d \sigma \\
& =\left(\rho\left(u_{N}\right)-\rho\left(\varphi_{N}\right)\right) T_{k}\left(u_{N}-\varphi_{N}\right) .
\end{aligned}
$$

As $\varphi_{N} \in L^{\infty}\left(\tilde{\Gamma}_{N e}\right)$, we obtain $\tilde{\rho}\left(\varphi_{N}\right) \in L^{\infty}\left(\tilde{\Gamma}_{N e}\right)$ and so $\tilde{\rho}\left(\varphi_{N}\right) \in L^{1}\left(\tilde{\Gamma}_{N e}\right)$ (as $\tilde{\Gamma}_{N e}$ is bounded) and by the Lebesgue dominated convergence theorem, we deduce that

$$
\lim _{\epsilon \rightarrow 0} \int_{\tilde{\Gamma}_{N e}} \tilde{\rho}\left(\varphi_{N}\right) T_{k}\left(u_{\epsilon}-\varphi_{N}\right) d \sigma=\int_{\tilde{\Gamma}_{N e}} \tilde{\rho}\left(\varphi_{N}\right) T_{k}\left(u_{N}-\varphi_{N}\right) d \sigma=\rho\left(\varphi_{N}\right) T_{k}\left(u_{N}-\varphi_{N}\right) .
$$

Hence,

$$
\limsup _{\epsilon \rightarrow 0} \int_{\tilde{\Gamma}_{N e}} \tilde{\rho}\left(u_{\epsilon}\right) T_{k}\left(u_{\epsilon}-\varphi_{N}\right) d \sigma \geq \rho\left(\varphi_{N}\right) T_{k}\left(u_{N}-\varphi_{N}\right) .
$$

Passing to the limit as $\epsilon \rightarrow 0$ in (4.1) and using (4.2)-(4.7), we see that $u$ is an entropy solution of $P(\rho, f, d)$.

We now prove the uniqueness part of Theorem 2.4.

Let $u$ and $v$ be two entropy solutions of $P(\rho, f, d)$. 
Let $h>0$. For $u$, we take $\xi=T_{h}(v)$ as test function and for $v$, we take $\xi=T_{h}(u)$ as test function in (2.9), to get for any $k>0$ with $k<h$,

$$
\left\{\begin{array}{l}
\int_{\Omega}\left(\sum_{i=1}^{N} a_{i}\left(x, \frac{\partial}{\partial x_{i}} u\right) \frac{\partial}{\partial x_{i}} T_{k}\left(u-T_{h}(v)\right)\right) d x+\int_{\Omega} b(u) T_{k}\left(u-T_{h}(v)\right) d x \leq \\
\int_{\Omega} f T_{k}\left(u-T_{h}(v)\right) d x+\left(d-\rho\left(u_{N e}\right) T_{k}\left(u_{N e}-T_{h}(v)\right)\right.
\end{array}\right.
$$

and

$$
\left\{\begin{array}{l}
\int_{\Omega}\left(\sum_{i=1}^{N} a_{i}\left(x, \frac{\partial}{\partial x_{i}} v\right) \frac{\partial}{\partial x_{i}} T_{k}\left(v-T_{h}(u)\right)\right) d x+\int_{\Omega} b(v) T_{k}\left(v-T_{h}(u)\right) d x \leq \\
\int_{\Omega} f T_{k}\left(v-T_{h}(u)\right) d x+\left(d-\rho\left(v_{N e}\right) T_{k}\left(v_{N e}-T_{h}(u)\right) .\right.
\end{array}\right.
$$

By adding (4.8) and (4.9), we obtain

$$
\begin{cases}\int_{\Omega}\left(\sum_{i=1}^{N} a_{i}\left(x, \frac{\partial}{\partial x_{i}} u\right) \frac{\partial}{\partial x_{i}} T_{k}\left(u-T_{h}(v)\right)\right) d x & \\ +\int_{\Omega}\left(\sum_{i=1}^{N} a_{i}\left(x, \frac{\partial}{\partial x_{i}} v\right) \frac{\partial}{\partial x_{i}} T_{k}\left(v-T_{h}(u)\right)\right) d x & :=A(h, k) \\ +\int_{\Omega} b(u) T_{k}\left(u-T_{h}(v)\right) d x+\int_{\Omega} b(v) T_{k}\left(v-T_{h}(u)\right) d x & :=B(h, k) \\ +\rho\left(u_{N e}\right) T_{k}\left(u_{N e}-T_{h}(v)\right)+\rho\left(v_{N e}\right) T_{k}\left(v_{N e}-T_{h}(u)\right) & :=C(h, k) \\ \leq \int_{\Omega} f T_{k}\left(u-T_{h}(v)\right) d x+\int_{\Omega} f T_{k}\left(v-T_{h}(u)\right) d x & :=D(h, k) \\ +d T_{k}\left(u_{N e}-T_{h}(v)\right)+d T_{k}\left(v_{N e}-T_{h}(u)\right) & :=E(h, k) .\end{cases}
$$

Let us introduce the following subsets of $\Omega$.

$$
\begin{aligned}
A_{0} & :=[|u-v|<k,|u|<h,|v|<h] \\
A_{1} & :=\left[\left|u-T_{h}(v)\right|<k,|v| \geq h\right] \\
A_{1}^{\prime} & :=\left[\left|v-T_{h}(u)\right|<k,|u| \geq h\right] \\
A_{2} & :=\left[\left|u-T_{h}(v)\right|<k,|u| \geq h,|v|<h\right] \\
A_{2}^{\prime} & :=\left[\left|v-T_{h}(u)\right|<k,|v| \geq h,|u|<h\right] .
\end{aligned}
$$

We have

$$
\begin{cases}A(h, k)= & :=I_{0}(h, k) \\ \int_{A_{0}}\left(\sum_{i=1}^{N}\left(a_{i}\left(x, \frac{\partial}{\partial x_{i}} u\right)-a_{i}\left(x, \frac{\partial}{\partial x_{i}} v\right)\right) \frac{\partial}{\partial x_{i}}(u-v)\right) d x & \\ +\int_{A_{1}}\left(\sum_{i=1}^{N} a_{i}\left(x, \frac{\partial}{\partial x_{i}} u\right) \frac{\partial}{\partial x_{i}} u\right) d x+\int_{A_{1}^{\prime}}\left(\sum_{i=1}^{N} a_{i}\left(x, \frac{\partial}{\partial x_{i}} v\right) \frac{\partial}{\partial x_{i}} v\right) d x & :=I_{1}(h, k) \\ +\int_{A_{2}}\left(\sum_{i=1}^{N} a_{i}\left(x, \frac{\partial}{\partial x_{i}} u\right) \frac{\partial}{\partial x_{i}}(u-v)\right) d x & \\ +\int_{A_{2}^{\prime}}\left(\sum_{i=1}^{N} a_{i}\left(x, \frac{\partial}{\partial x_{i}} v\right) \frac{\partial}{\partial x_{i}}(v-u)\right) d x & :=I_{2}(h, k) .\end{cases}
$$


The term $I_{1}(h, k)$ is non-negative since each term in $I_{1}(h, k)$ is non-negative.

For the term $I_{2}(h, k)$, as

$$
I_{2}(h, k)+\int_{A_{2}}\left(\sum_{i=1}^{N} a_{i}\left(x, \frac{\partial}{\partial x_{i}} u\right) \frac{\partial}{\partial x_{i}} v\right) d x+\int_{A_{2}^{\prime}}\left(\sum_{i=1}^{N} a_{i}\left(x, \frac{\partial}{\partial x_{i}} v\right) \frac{\partial}{\partial x_{i}} u\right) d x=I_{1}(h, k),
$$

so,

$$
I_{2}(h, k) \geq-\left(\int_{A_{2}}\left(\sum_{i=1}^{N} a_{i}\left(x, \frac{\partial}{\partial x_{i}} u\right) \frac{\partial}{\partial x_{i}} v\right) d x+\int_{A_{2}^{\prime}}\left(\sum_{i=1}^{N} a_{i}\left(x, \frac{\partial}{\partial x_{i}} v\right) \frac{\partial}{\partial x_{i}} u\right) d x\right) .
$$

Let us show that $-\left(\int_{A_{2}}\left(\sum_{i=1}^{N} a_{i}\left(x, \frac{\partial}{\partial x_{i}} u\right) \frac{\partial}{\partial x_{i}} v\right) d x\right)$ goes to 0 as $h \rightarrow \infty$.

We have

$$
\left\{\begin{array}{l}
\left|\int_{A_{2}}\left(\sum_{i=1}^{N} a_{i}\left(x, \frac{\partial}{\partial x_{i}} u\right) \frac{\partial}{\partial x_{i}}(v)\right) d x\right| \leq \\
C \sum_{i=1}^{N}\left(\left|j_{i}\right|_{p_{i}^{\prime}(.)}+\left|\frac{\partial u}{\partial x_{i}}\right|_{L^{p_{i}(.)}(\{h<|u| \leq h+k\})}^{p_{i}(x)-1}\right)\left|\frac{\partial v}{\partial x_{i}}\right|_{L^{p_{i}(.)}(\{h-k<|v| \leq h\})}
\end{array} .\right.
$$

For all $i=1, \ldots N$, the quantity $\left(\left|j_{i}\right|_{p_{i}^{\prime}(.)}+\left|\frac{\partial u}{\partial x_{i}}\right|_{L^{p_{i}(.)}(\{h<|u| \leq h+k\})}^{p_{i}(x)-1}\right)$ is finite since

$u=T_{h+k}(u) \in \mathcal{T}_{N e}^{1, \vec{p}(.)}(\Omega)$ and $j_{i} \in L^{p_{i}^{\prime}(.)}(\Omega)$; then by Lemma 3.4, the last expression converges to zero as $h$ tends to infinity.

Similarly we can show that $-\left(\int_{A_{2}}\left(\sum_{i=1}^{N} a_{i}\left(x, \frac{\partial}{\partial x_{i}} v\right) \frac{\partial}{\partial x_{i}}(u)\right) d x\right)$ goes to 0 as $h \rightarrow \infty$, hence, we obtain

$$
\limsup _{h \rightarrow \infty} A(h, k) \geq \int_{[|u-v|<k]}\left[\sum_{i=1}^{N}\left(a_{i}\left(x, \frac{\partial}{\partial x_{i}} u\right)-a_{i}\left(x, \frac{\partial}{\partial x_{i}} v\right)\right) \frac{\partial}{\partial x_{i}}(u-v)\right] d x .
$$

By using the Lebesgue dominated convergence theorem, it yields that

$$
\lim _{h \rightarrow \infty} B(h, k)=\int_{\Omega}(b(u)-b(v)) T_{k}(u-v) d x \text { and } \lim _{h \rightarrow \infty} D(h, k)=0 .
$$

For $h$ large enough, we get

$$
\lim _{h \rightarrow \infty} C(h, k)=\left(\rho\left(u_{N}\right)-\rho\left(v_{N}\right)\right) T_{k}\left(u_{N}-v_{N}\right) \text { and } \lim _{h \rightarrow \infty} E(h, k)=0 .
$$

Letting $h$ goes to $\infty$ in (4.10) and combining (4.12)-(4.13), we obtain

$$
\left\{\begin{array}{l}
\int_{[|u-v|<k]}\left[\sum_{i=1}^{N}\left(a_{i}\left(x, \frac{\partial}{\partial x_{i}} u\right)-a_{i}\left(x, \frac{\partial}{\partial x_{i}} v\right)\right) \frac{\partial}{\partial x_{i}}(u-v)\right] d x \\
+\int_{\Omega}(b(u)-b(v)) T_{k}(u-v) d x+\left(\rho\left(u_{N}\right)-\rho\left(v_{N}\right)\right) T_{k}\left(u_{N}-v_{N}\right) \leq 0 .
\end{array}\right.
$$

All the terms in the left hand side of (4.14) are non-negative so that we get $\forall k>0$,

$$
\int_{[|u-v|<k]}\left[\sum_{i=1}^{N}\left(a_{i}\left(x, \frac{\partial}{\partial x_{i}} u\right)-a_{i}\left(x, \frac{\partial}{\partial x_{i}} v\right)\right) \frac{\partial}{\partial x_{i}}(u-v)\right] d x=0
$$

and

$$
\left\{\begin{array}{l}
\int_{\Omega}(b(u)-b(v)) T_{k}(u-v) d x=0 \\
\left(\rho\left(u_{N}\right)-\rho\left(v_{N}\right)\right) T_{k}\left(u_{N}-v_{N}\right)=0
\end{array}\right.
$$


Relation (4.15) gives $\frac{\partial}{\partial x_{i}}(u-v)=0$ a.e. in $\Omega$; we deduce that there exists a constant $c$ such that $u-v=c$ a.e. in $\Omega$. We deduce from (4.16) $b(u)=b(v)=0$ a.e. in $\Omega$. As $b$ is invertible,

$$
\left\{\begin{array}{l}
u=v \text { a.e. in } \Omega \\
\rho\left(u_{N}\right)=\rho\left(v_{N}\right)
\end{array}\right.
$$

which prove the uniqueness part.

\section{REFERENCES}

[1] M. Bendahmane and K. H. Karlsen; Anisotropic nonlinear elliptic systems with measure data and anisotropic harmonic maps into spheres. Electron. J. Differ. Equ. 2006 (2006), 46.

[2] Ph. Bénilan, H. Brézis and M. G. Crandall; A semilinear equation in $L^{1}\left(\mathbb{R}^{N}\right)$. Ann. Scuola. Norm. Sup. Pisa, 2 (1975), $523-555$.

[3] B. K. Bonzi, S. Ouaro and F. D. Y. Zongo; Entropy solution for nonlinear elliptic anisotropic homogeneous Neumann Problem, Int. J. Differ. Equ. 2003 (2013), Article ID 476781.

[4] M. M. Boureanu and V. D. Radulescu; Anisotropic Neumann problems in Sobolev spaces with variable exponent. Nonlinear Anal. TMA 75 (12) (2012), 4471-4482.

[5] Y. Ding, T. Ha-Duong, J. Giroire, V. Moumas, Modeling of single-phase flow for horizontal wells in a stratified medium. Comput. Fluids, 33 (2004), 715-727.

[6] J. Giroire, T. Ha-Duong, V. Moumas, A non-linear and non-local boundary condition for a diffusion equation in petroleum engineering. Math. Methods Appl. Sci. 28 (13) (2005), 1527-1552.

[7] P. Halmos, Measure Theory, D. Van Nostrand Company, New York, 1950.

[8] B. Koné, S. Ouaro and F. D. Y. Zongo; Nonlinear elliptic anisotropic problem with fourier boundary condition , Int. J. Evol. Equ. 8 (4) (2013), 305-328.

[9] X. Fan and D. Zhao; On the spaces $L^{p(.)}(\Omega)$ and $W^{m, p(.)}(\Omega)$. J. Math. Anal. Appl. 263 (2001), 424-446.

[10] X. Fan; Anisotropic variable exponent Sobolev spaces and $\vec{p}($.$) -Laplacian equations. Complex Var. Elliptic Equ. 56$ (7-9) (2011), 623-642.

[11] M. Sanchon and J. M. Urbano; Entropy solutions for the $p(x)$-Laplace Equation. Trans. Amer. Math. Soc. 361 (12) (2009), $6387-6405$.

[12] M. Troisi; teoremi di inclusione per spazi di Sobolev non isotropi. Ric. Mat. 18 (1969), 3-24.

[13] R. E. Showalter; Monotone operators in Banach space and nonlinear partial differential equations, Mathematical Surveys and Monographs, vol. 49, American Mathematical Society, Providence, RI, 1997. 